\title{
Influence of Finite Span and Sweep on Active Flow Control Efficacy
}

\author{
David Greenblatt* \\ Technical University of Berlin, Berlin, Germany \\ Anthony E. Washburn ${ }^{\dagger}$ \\ NASA Langley Research Center, Hampton VA 23681-2199
}

\begin{abstract}
Active flow control efficacy was investigated by means of leading-edge and flap-shoulder zero mass-flux blowing slots on a semispan wing model that was tested in unswept (standard) and swept configurations. On the standard configuration, stall commenced inboard, but with sweep the wing stalled initially near the tip. On both configurations, leading-edge perturbations increased $C_{L, \max }$ and post stall lift, both with and without deflected flaps. Without sweep, the effect of control was approximately uniform across the wing span but remained effective to high angles of attack near the tip; when sweep was introduced a significant effect was noted inboard, but this effect degraded along the span and produced virtually no meaningful lift enhancement near the tip, irrespective of the tip configuration. In the former case, control strengthened the wingtip vortex; in the latter case, a simple semi-empirical model, based on the trajectory or "streamline" of the evolving perturbation, served to explain the observations. Control on finite-span flaps did not differ significantly from their two-dimensional counterpart, while control over a tip flap produced significant variations to all three moments in the presence of large deflection and these variations were linear with input slot momentum. Control from the flap produced expected lift enhancement and $C_{L, \max }$ improvements in the absence of sweep, but these improvements degraded with the introduction of sweep.
\end{abstract}

\section{Nomenclature}

$\begin{array}{ll}A R & =\text { wing model aspect ratio } \\ c & =\text { model chord-length } \\ C_{d p} & =\text { sectional form-drag coefficient } \\ C_{D p} & =\text { model form-drag coefficient } \\ C_{l} & =\text { sectional lift coefficient } \\ C_{L} & =\text { model lift coefficient } \\ C_{m} & =\text { sectional moment coefficient } \\ C_{M} & =\text { model pitching moment coefficient } \\ C_{R} & =\text { model rolling moment coefficient } \\ C_{Y} & =\text { model yawing moment coefficient } \\ C_{p} & =\text { time-mean pressure coefficient } \\ C_{\mu} & =\text { slot momentum coefficient, } h / c\left(U_{j} / U_{\infty}\right)^{2} \\ f & =\text { separation control excitation frequency } \\ F^{+} & =\text {reduced excitation frequency, } f X / U_{\infty} \\ h & =\text { slot width } \\ L_{f} & =\text { flap length, from slot to trailing-edge } \\ q & =\text { free-stream dynamic pressure } \\ R e & =\text { Reynolds number based on chord length }\end{array}$

\footnotetext{
* Senior Research Scientist. Present Address: Technical University of Berlin, 8 Mueller Breslau Street, D-10623 Berlin, Germany. Senior Member. david.greenblatt@pi.tu-berlin.de.

${ }^{\dagger}$ Branch Head, Flow Physics and Control Branch. Senior Member. Anthony.E.Washburn@nasa.gov 


$\begin{array}{ll}s & =\text { wing semi-span length, } b / 2 \\ U_{j} & =\text { peak jet slot blowing velocity } \\ U_{\infty} & =\text { free-stream velocity } \\ U_{\phi} & =\text { phase velocity of the fundamental pressure perturbation } \\ U, V, W & =\text { mean velocities in directions } x, y, z \\ X & =\text { distance from perturbation to wing trailing-edge } \\ x, y, z & =\text { coordinates measured from model leading-edge and root (left-hand system) } \\ \alpha & =\text { angle of attack } \\ \alpha_{s} & =\text { static stall angle } \\ \delta & =\text { flap deflection angles, }\left(\delta_{i}, \delta_{o}, \delta_{t}\right) \\ \Lambda & =\text { sweep angle } \\ \text { Subscripts } & =\text { inboard } \\ i & =\text { flap-shoulder } \\ f S & =\text { leading-edge } \\ l e & =\text { normal to the leading-edge } \\ n & =\text { tip, tangential to the leading-edge } \\ o & =\text { trailing-edge } \\ t & \text { swept wing } \\ t e & \end{array}$

\section{Introduction}

$\mathrm{T}$

he capability and limitations of zero mass-flux active flow control (AFC) are reasonably well understood in relatively simple two-dimensional flows and on airfoils. [1] Thus for high aspect ratio unswept wings, first order performance approximations can be made directly. However, for low aspect ratio wings - for example on combat, unmanned and micro vehicles, and control surfaces - significant three-dimensional effects preclude direct extensions of two-dimensional results. Currently very little is known of the efficacy of AFC in conjunction with three-dimensional effects. Nevertheless, some basic understanding has been attained on a yawed airfoil that approximated infinite span, [2] where it was shown that standard sweep transformations were appropriate for analyzing active flow control data. A number of AFC investigations have also been carried out on delta wings and highly swept planforms. [3-6] Control extended vortex breakdown by about $25 \%$, thereby generating increased lift. $[3,4]$ Control by pulse-modulation of high frequency piezo-electric actuators generated maximum lift at $F^{+}=\mathrm{O}(1)$. [5] PIV measurements suggested that the pulsing enhances momentum transfer across the shear layer, downstream of the original vortex breakdown location, and generates a streamwise vortex whose size is commensurate with the local wingspan.

Presently, very little is known about the efficacy of control near wingtips and flap edges, where the flow becomes strongly three-dimensional due to an abrupt change in the spanwise geometry. These tip and edge flows become further complicated with the introduction of sweep. In this paper an attempt was made to systematically study threedimensional effects by designing and testing an active flow control semispan model in unswept (standard) and swept configurations. The model was equipped a leading-edge slot and three identical simple flaps with individual control slots. The model could also incorporate arbitrary wingtip designs. The overall strategy was to introduce successively larger three-dimensional effects, starting with the relatively innocuous unswept configuration, followed by individual flap deflections, the introduction of sweep and finally the introduction of sweep combined with flap deflections. Control was introduced via zero mass-flux blowing slots at the leading-edge and flap-shoulder. Surface pressure ports, integrated to yield wing loads, were used to gauge the efficacy of control. Limited flowfield measurements were made above the flaps and in the wake of the model.

\section{Experimental Setup \& Testing}

\section{A. Semispan Model Description}

Low speed AFC experiments were performed on a rectangular planform semispan NACA 0015 model ( $A R=4$; semispan $s=609.6 \mathrm{~mm}$, chord $c=304.8 \mathrm{~mm})$. The model incorporated three identical simple flaps of span $s_{f}=1 / 3 s$ hinged at $0.7 c$ (inboard, outboard and tip; fig. 1a) and was equipped with leading-edge and flap-shoulder flow 
control slots, joined to interior plenums (fig. 1b). Flaps were deflected in unison and also deflected individually to assess the effects of three-dimensionality. The notation $\delta \equiv\left(\delta_{i}, \delta_{o}, \delta_{t}\right)$ is used throughout to indicate the particular configuration under consideration. Forcing was supplied to the plenums via Aero and Thermally Engineered Actuator Modules (ATEAM) voice-coil based actuators. ${ }^{*}$ The zero mass-flux control slot velocities were calibrated using a hot wire along the span of the wing. The perturbation amplitude uncertainty was estimated at $\Delta C_{\mu} / C_{\mu} \leq 20 \%$. The model was equipped with 165 static pressure ports arranged in a perpendicular spanwise and chordwise grid (fig. 1c), with additional rows of pressure ports on the flaps, and these surface pressures were integrated to estimate aerodynamic loads. The model was also equipped with unsteady pressure transducers: nine were mounted on the wing upper surface and three are mounted within the wing plenums to monitor control frequencies and amplitudes. Steady and unsteady wing surface pressure data were acquired for angles of attack $\alpha \leq 33^{\circ}$ and flap deflection angles of $0^{\circ} \leq \delta \leq 40^{\circ}$. Wing static pressures were measured using a high-speed pressure scanner and unsteady pressures were measured by means of piezoresistive unsteady pressure transducers. The main source of error in the pressure measurements was due to precision, with $C_{p} \leq \pm 0.02$, based on $95 \%$ confidence intervals.

The wing was tested in both a "standard" (unswept) configuration (see figs. 1a-1c; photograph in fig. 1d) and a "swept" configuration (see figs. 2a-2c; photograph in fig. 2d), where control perturbations were supplied from both slots. Sweep was achieved by means of a triangular wedge, also with a NACA 0015 profile, which was also instrumented with pressure ports (figs. 2a-2c). Furthermore, various tip extensions were tested, namely: (a) "no tip extension" and (b) a square tip extension for the standard configuration (figs. 1a and 1b respectively); and (a) "no tip extension," (b) square tip and (c) parallel tip extensions for the swept configuration (figs. 2a-2c respectively). The wingtip extensions were not instrumented with pressure ports. In the swept configuration, flap deflection resulted in a gap between the inboard edge of the inboard flap and the tunnel wall (see photograph in fig. $2 \mathrm{~d}$, where all flaps are deflected to $20^{\circ}$ ). A removable fairing was employed to seal the gap and its effect on control effectiveness was also considered.

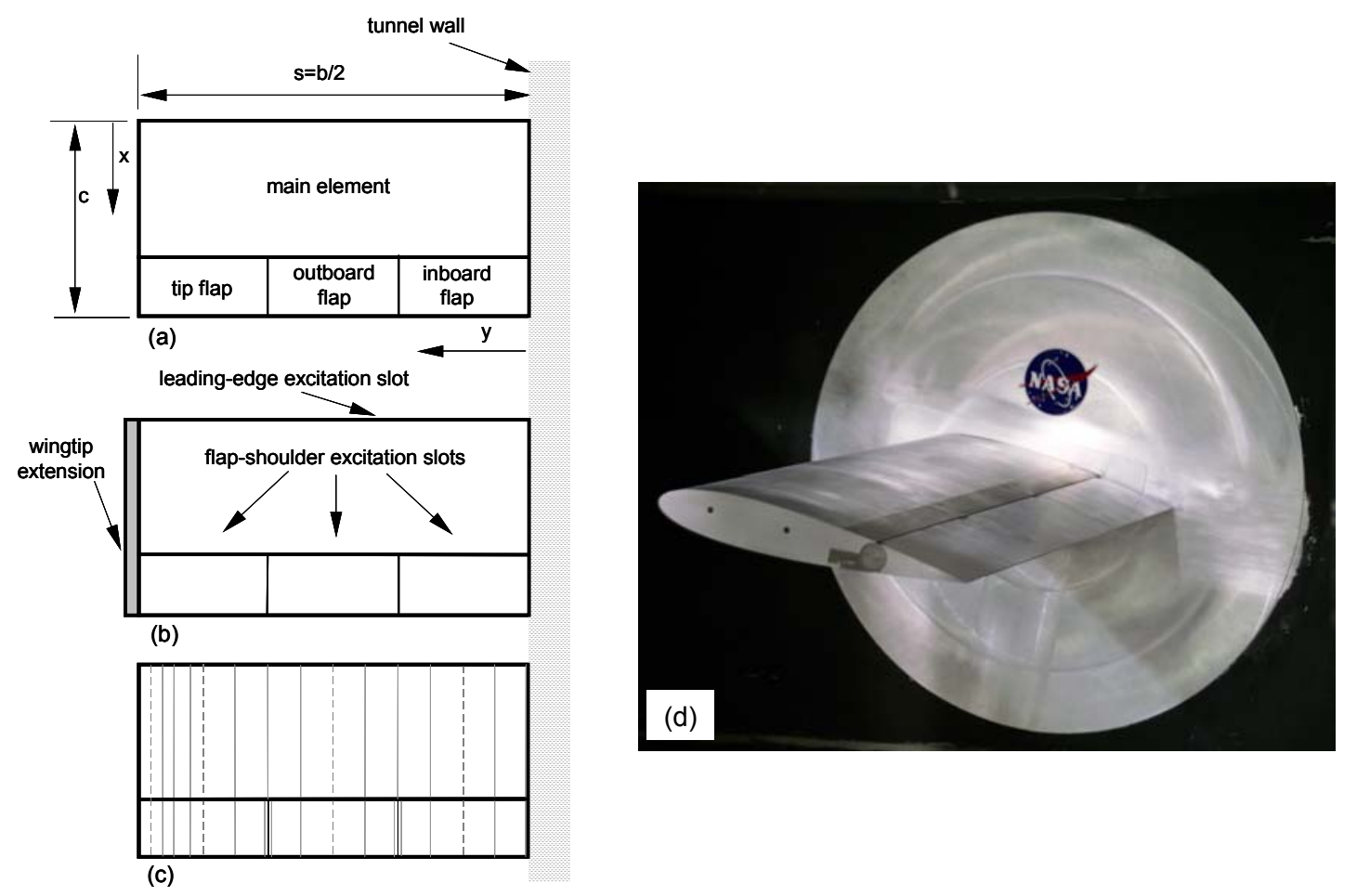

Fig. 1. Schematics of the unswept (standard) semispan configuration, showing (a) wing components and lefthand coordinate system with no tip extension; (b) locations of the control slots and the wingtip extension; (c) locations of the pressure ports on the main-element and flap (d) photograph of the unswept semi-span wing in the Basic Aerodynamics Research Tunnel (without the wingtip extension).

\footnotetext{
\$ Nagib, H., Kiedaisch, J., Wygnanski, I., Stalker, A, Wood, T., and McVeigh, M., "First-In-Flight Full-Scale Application of Active Flow Control: The XV-15 Tiltrotor Download Alleviation", NATO RTO-MP-AVT-111-P-29, NATO AVT-111/RSM, Prague, Czech Republic, October 2004.
} 

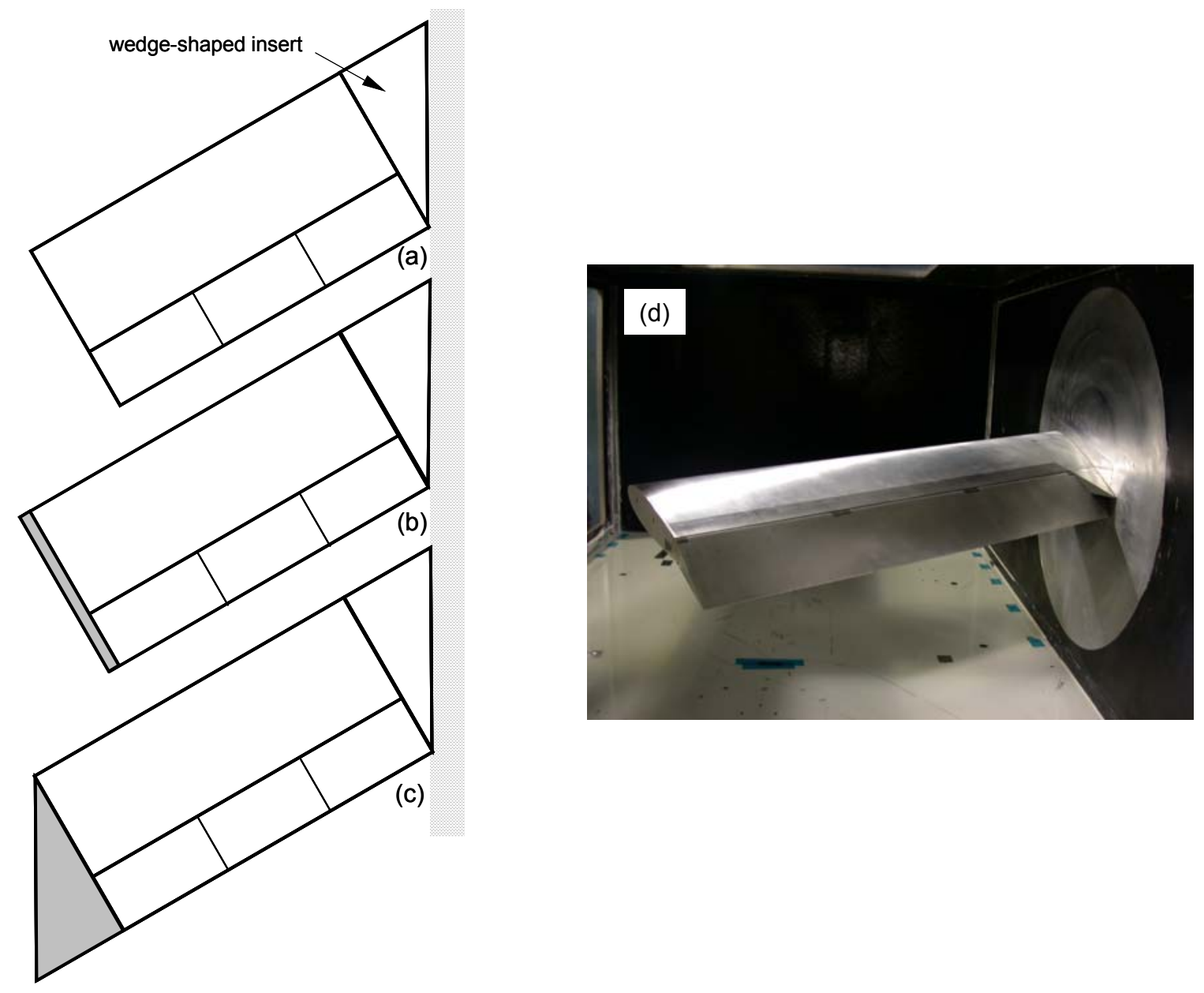

Fig. 2. Schematics of the swept semispan configuration, showing (a) no wingtip extension; (b) the square wingtip extension; and (c) the parallel wingtip extension (d) photograph of the swept semi-span wing in the Basic Aerodynamics Research Tunnel (without wingtip extensions).

\section{B. Data Reduction}

With the introduction of sweep, pressure data were analyzed with respect to the velocity component normal to the wing leading-edge (cf. [2]). This facilitated a direct comparison of corresponding surface pressures for the standard and swept configurations. Since the wing is cantilevered, the infinite sweep relations are modified to account for the variable angle of attack, thus:

$$
\Lambda^{\prime}=\arctan (\tan \Lambda \cos \alpha)
$$

and hence the component of velocity normal to the leading is

$$
U_{\infty, n}=U_{\infty} \cos \Lambda^{\prime} .
$$

Substituting this relation into the definition for two-dimensional pressure coefficient, gives

$$
C_{p, n}=C_{p, s w} / \cos ^{2} \Lambda^{\prime}
$$

The AFC parameters were also modified to account for the cantilevered and swept wing, thus:

$$
\begin{aligned}
& C_{\mu, n}=C_{\mu, s w} / \cos ^{2} \Lambda^{\prime} \\
& F_{n}^{+}=F_{s w}^{+} / \cos \Lambda^{\prime}
\end{aligned}
$$


For convenience and brevity the subscript $n$ in equations (2)-(5) is dropped from the remaining parameters in the following discussions, but is always implied for the swept configuration. The definition of $\Lambda^{\prime}$ in eqn. (1) above, requires a variation in $f$ and $U_{\infty}$ at every $\alpha$ in order to maintain constant $C_{\mu, n}$ and $F_{n}^{+}$, but this did not prove to be practical. Therefore, these parameters are always cited for $\alpha=0^{\circ}$ and it is understood that they are over-predicted by maximum values of $8 \%$ and $4 \%$ respectively at $\alpha=33^{\circ}$.

\section{Three-Dimensional Configurations \& Strategy}

In an attempt to introduce three-dimensional effects gradually, a graded approach was adopted, and the study was divided into five categories. A: standard and swept configurations with leading-edge control, with and without flap deflections; B: combined control from the leading-edge and flap shoulder (discussed in ref. [7]); C and D: the deflection of individual flaps with control at the shoulder; and E: sweep combined with flap shoulder control. More details regarding the configurations and section references can be found in table 1 .

Table 1. Details of the different configurations tested in the present study.

\begin{tabular}{|c|c|c|c|c|c|}
\hline Cat. & $\left(\delta_{i}, \delta_{o}, \delta_{t}\right)$ & control location & wing configuration & tip configuration & section/ref. \\
\hline \hline A & $\left(0^{\circ}, 0^{\circ}, 0^{\circ}\right)$ & leading-edge & standard/swept & $\begin{array}{c}\text { no extension/with } \\
\text { extension/parallel tip }\end{array}$ & $3.1 .1-3.1 .4$ \\
\hline A & $\left(20^{\circ}, 20^{\circ}, 20^{\circ}\right)$ & leading-edge & standard/swept & $\begin{array}{c}\text { no extension/with } \\
\text { extension/parallel tip }\end{array}$ & 3.1 .5 \\
\hline A & $\left(40^{\circ}, 40^{\circ}, 40^{\circ}\right)$ & leading-edge & standard/swept & $\begin{array}{c}\text { no extension/with } \\
\text { extension/parallel tip }\end{array}$ & 3.1 .5 \\
\hline B & $\left(20^{\circ}, 20^{\circ}, 0^{\circ}\right)$ & combined & Standard & $\begin{array}{c}\text { no extension/ } \\
\text { with extension }\end{array}$ & ref. [7] \\
\hline B & $\left(20^{\circ}, 20^{\circ}, 20^{\circ}\right)$ & combined & Standard & $\begin{array}{c}\text { no extension/ } \\
\text { with extension }\end{array}$ & ref. [7] \\
\hline B & $\left(40^{\circ}, 40^{\circ}, 40^{\circ}\right)$ & combined & Standard & $\begin{array}{c}\text { no extension/ } \\
\text { with extension }\end{array}$ & ref. [7] \\
\hline C & $\left(20^{\circ}, 20^{\circ}, 0^{\circ}\right)$ & flap-shoulder & standard/swept & $\begin{array}{c}\text { no extension } \\
\text { no extension }\end{array}$ & 3.2 .1 \\
\hline C & $\left(20^{\circ}, 0^{\circ}, 0^{\circ}\right)$ & flap-shoulder & standard/swept & no extension & 3.2 .1 \\
\hline C & $\left(0^{\circ}, 20^{\circ}, 0^{\circ}\right)$ & flap-shoulder & standard/swept & $\begin{array}{c}\text { no extension/ } \\
\text { with extension }\end{array}$ & 3.2 .2 \\
\hline D & $\left(0^{\circ}, 0^{\circ}, 20^{\circ}\right)$ & flap-shoulder & standard/swept & $\begin{array}{c}\text { no extension/ } \\
\text { with extension }\end{array}$ & 3.2 .2 \\
\hline D & $\left(0^{\circ}, 0^{\circ}, 40^{\circ}\right)$ & flap-shoulder & standard/swept & $\begin{array}{c}\text { no extension/with } \\
\text { extension/parallel tip }\end{array}$ & 3.2 .3 \\
\hline E & $\left(20^{\circ}, 20^{\circ}, 20^{\circ}\right)$ & flap-shoulder & swept & $\begin{array}{c}\text { no extension/with } \\
\text { extension/parallel tip }\end{array}$ & 3.2 .3 \\
\hline E & $\left(40^{\circ}, 40^{\circ}, 40^{\circ}\right)$ & flap-shoulder & swept & &
\end{tabular}

\section{Discussion of Results}

\section{A. Stall Mechanism and Leading-edge Control}

Preliminary data were acquired at $R e=500,000$ and $R e=1,000,000$ for the standard (unswept) configuration without flap deflections or wingtips. Differences between the two data sets were minor because the leading-edge slot effectively tripped the boundary layer and the sharp square wingtip fixed separation on the lower edge of the wingtip independent of Re. Baseline and controlled wing lift coefficient data are shown for both standard and swept configurations (fig. 3), with no wingtip extension installed (cf. schematics in figs. 1a and 2a). For both configurations, aerodynamic coefficients were based on the rectangular part of the planform only, i.e. pressure measurements on the wedge-shaped insert were not included in the sweep lift calculations. In both instances, leading-edge control was applied at $F^{+}=0.65$ and $C_{\mu}=0.3 \%$, although the physical frequencies and jet amplitudes were different to account for sweep (see eqns. 4 and 5). The choice of this reduced frequency was based on previous findings relating to effective frequencies on a NACA0015 airfoil [12,10] and confirmed on the present model. [7] 
Due to limitations associated with the $\alpha$ traversing system, data were acquired in the ranges $-4^{\circ} \leq \alpha \leq 17^{\circ}$ and $15^{\circ} \leq$ $\alpha \leq 33^{\circ}$. In the lower range, data at even angles are for $\alpha$ increasing and odd angles are for $\alpha$ decreasing and vice versa in the higher range. This convention was applied consistently throughout this paper.

The standard baseline configuration appears to stall at $\alpha \approx 18^{\circ}$ and exhibits significant post-stall hysteresis (a bi-stable flow). The application of leading-edge control effectively eliminates hysteresis, which is routinely observed on twodimensional (airfoil) configurations, and $C_{L, \max }$ is attained at $\alpha \approx 25^{\circ}$. The swept baseline configuration stalls very gently at $\alpha>20^{\circ}$ and, in contrast, shows very little hysteresis. With the application of control, the wing continuously generates lift with $\alpha$, albeit at low $d C_{L} / d \alpha$, and appears to be still increasing at $\alpha>33^{\circ}$ (maximum tested here). For both configurations, control increases $C_{L, \max }$ by approximately 0.23 . Due to the partially stalled nature of the flow, however, these post stall increases are accompanied by large drag increases.
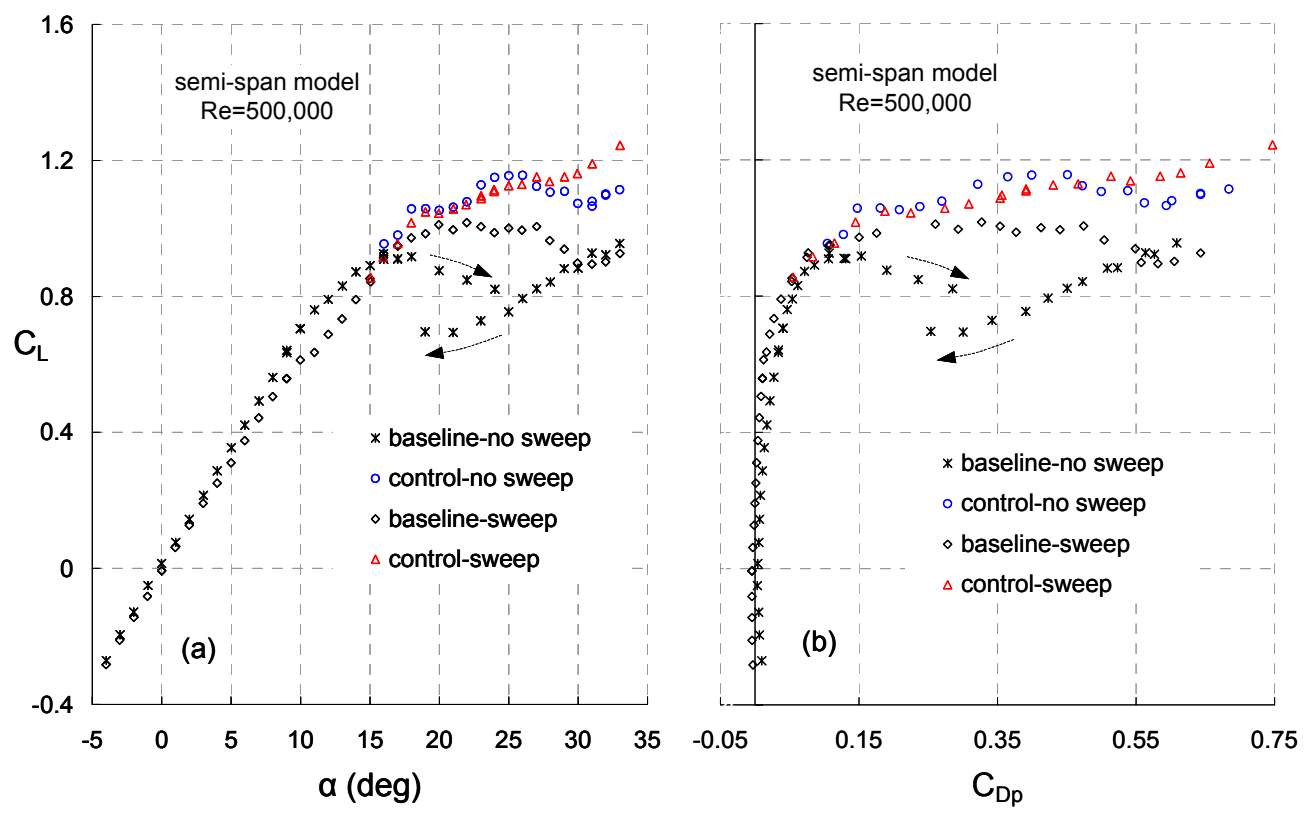

Fig. 3. Wing lift and form-drag coefficients based on integrated pressures on the rectangular section of the model (see fig. 1c). (No wingtip extensions.) Leading edge control at $F^{+}=0.65$ and $C_{\mu}=0.3 \%$.

\section{Comparison of Baseline Stall Mechanisms}

A preliminary assessment of the spanwise stall mechanisms for both configurations can be made by considering figs. $4 \mathrm{a}$ and $4 \mathrm{~b}$ respectively. For the standard configuration, incipient stall is evident inboard, close to the tunnel wall, at $\alpha=14^{\circ}$. However, the loss of lift inboard, evident for $\alpha>14^{\circ}$, is accompanied by an increase in lift outboard, and this continues with increasing angle of attack (fig. 4a). The distortion of the load distribution near the tip of the wing $(y / s>0.97)$ is due to the formation of vortex at the wingtip and these measurements are consistent with data of other investigations (e.g. [8,9]) at higher Reynolds numbers. Inboard stall, accompanied by continued outboard lift increases, results in an overall lift increase and the perceived wing stall is at $\alpha \approx 18^{\circ}$ (fig. 3a). With sweep, significantly more lift is generated inboard, but this is offset by the poor outboard lift generation (fig. 4b). Between $\alpha=17^{\circ}$ and $19^{\circ}$, changes to lift are negligible near the tip and stall is observed at $\alpha \approx 21^{\circ}$. Thus, when compared to the unswept case, the stall mechanism is reversed with lift continuously increasing inboard. The partial stall mechanisms (inboard for the standard configuration and outboard for sweep), when integrated over the span, manifests as the relatively gentle wing stall observed in figs. $3 a$ and $3 b$.

More evidence of the stall mechanism is provided by the surface pressure coefficient data shown for $\alpha=14^{\circ}$ at selected spanwise locations $(y / s=0.17,0.5,0.83,0.99)$ in figs. 5a to $5 \mathrm{~d}$ respectively. Inboard, the pressure distributions near the leading-edge indicate a transition bubble, in both the swept and unswept cases; thus it seems that here sweep does not result in a different transition mechanism. The pressure recovery associated with the standard configuration indicates incipient stall near the trailing-edge, consistent with NACA 0015 airfoil data, [10] 
while that of the swept configuration is consistent with attached flow. Further outboard, this situation reverses (see figs. $5 \mathrm{~b}$ and $5 \mathrm{c}$ and inset in fig. $5 \mathrm{~d}$ showing the trailing-edge $C_{p}$ ) and the swept trailing-edge indicates incipient stall outboard. The relatively low pressure at the tip of the standard configuration is due to the vortex rollup which is partially completed on the wingtip itself (cf. fig. 5d).
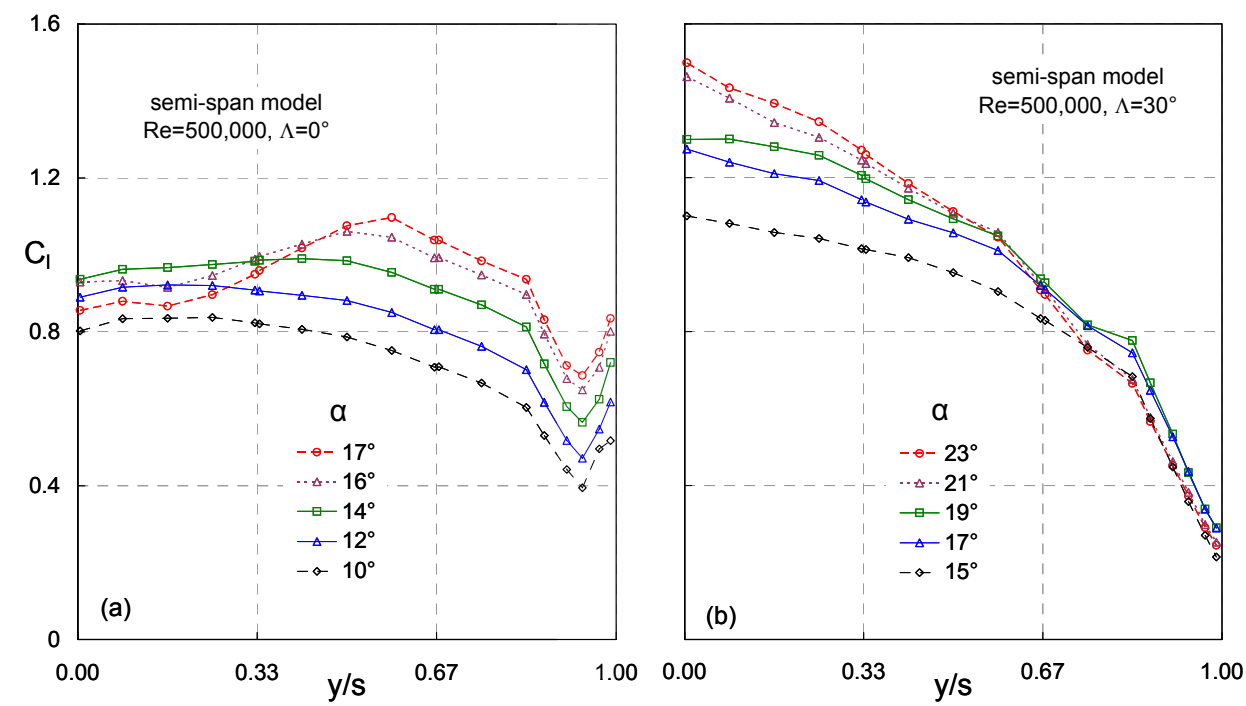

Fig. 4. Baseline span-load variations for (a) standard and (b) swept configurations based on integrated chordwise pressures on the rectangular section of the model corresponding to fig. 3. (No wingtip extensions.)
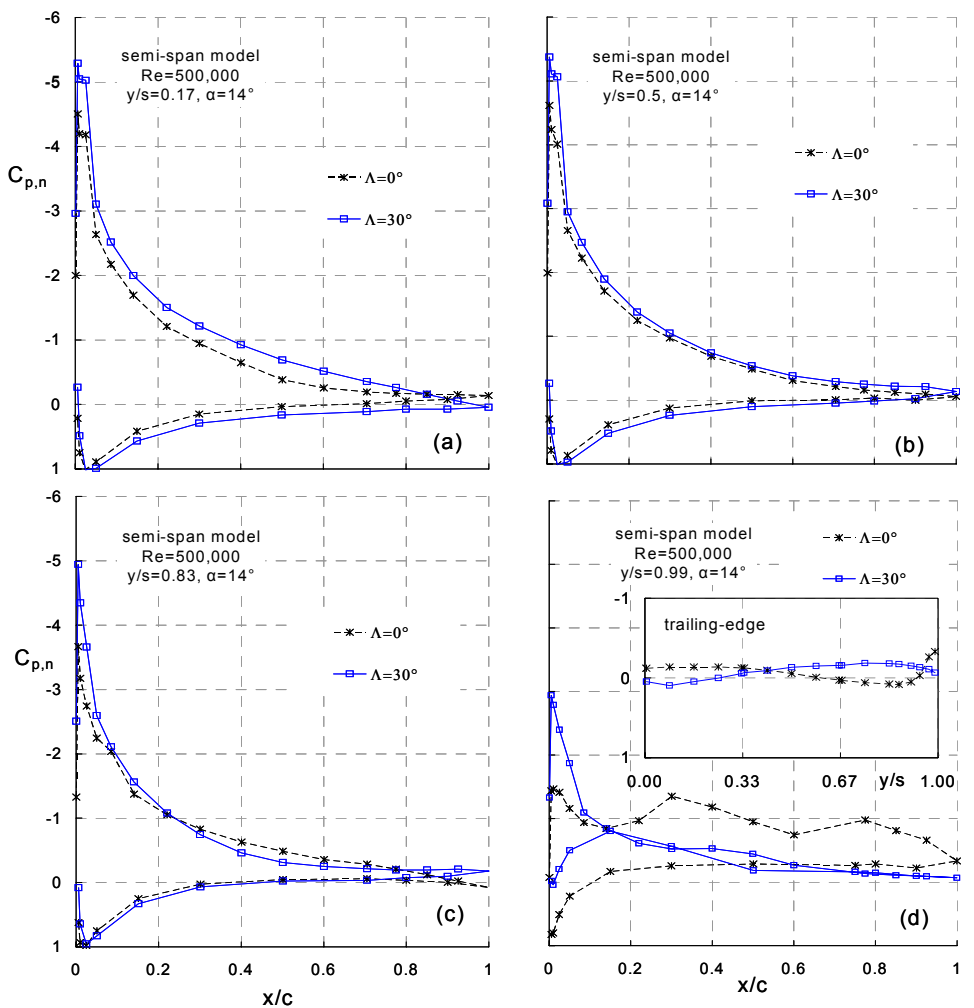

Fig. 5. Baseline pressure coefficient distributions at selected locations on the rectangular section of the model for standard and swept configurations (no wingtip extensions). Inset: corresponding trailing-edge pressure coefficients. 

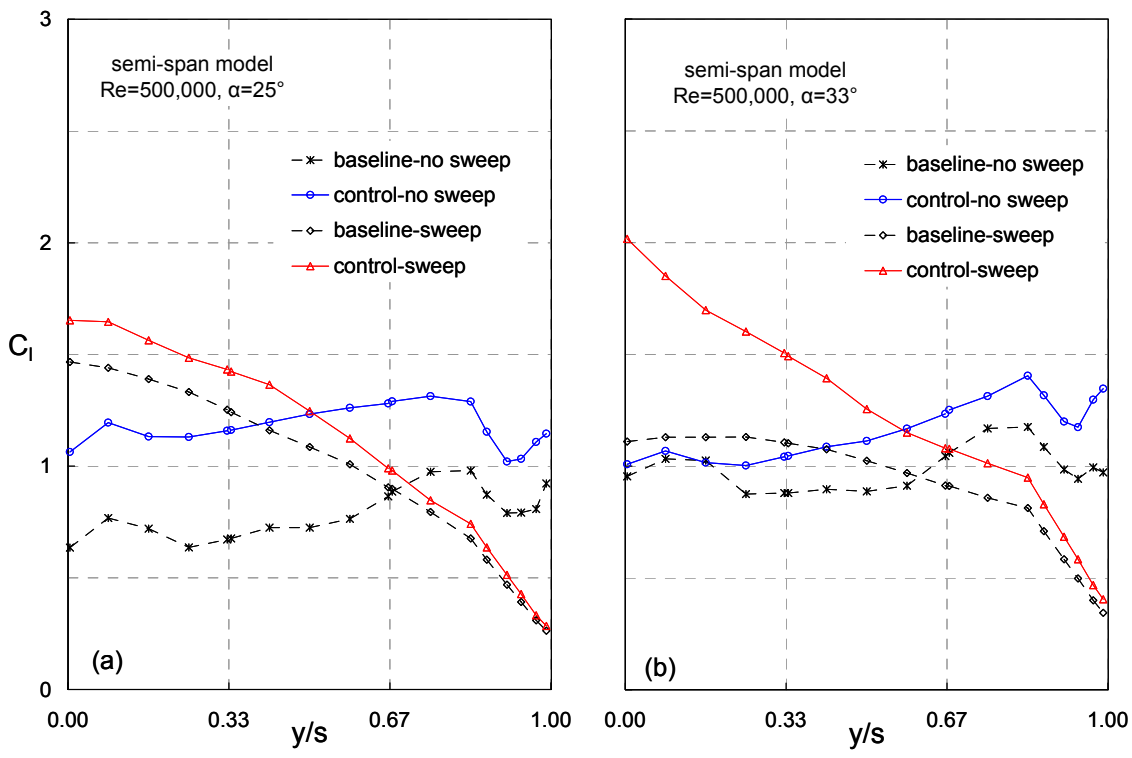

Fig. 6. Lift coefficient distributions, showing the effect of leading-edge control, at two angles of attack for both standard and swept configurations (no wingtip extension.) Control at $C_{\mu}=0.3 \%$ and $F^{+}=0.65$.
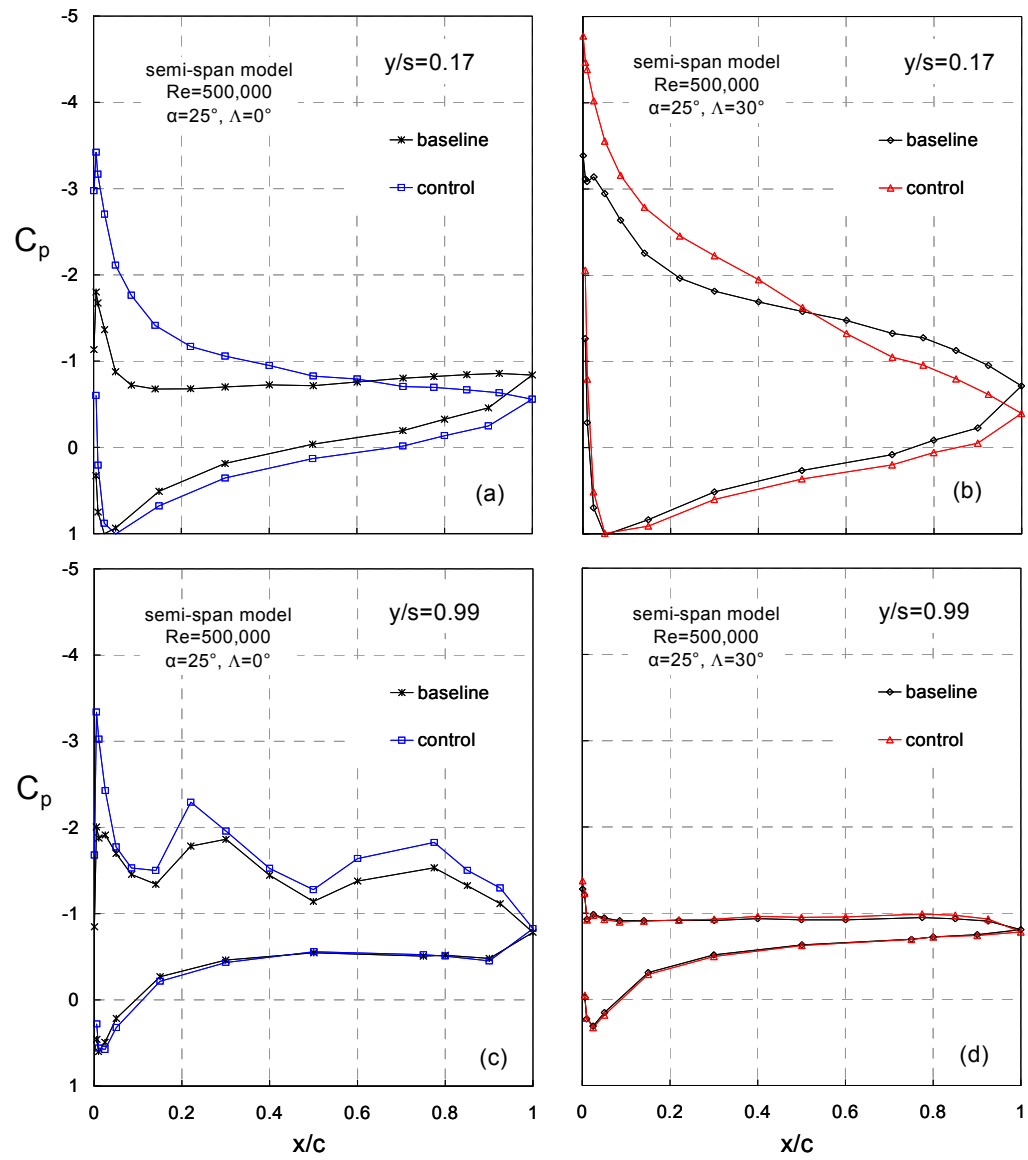

Fig. 7. Baseline and controlled pressure coefficient distributions at selected locations on the rectangular section of the model for standard and swept configurations $\left(\alpha=25^{\circ}\right.$; no wingtip extension). 


\section{Effect of Sweep on Leading-edge Control}

Figs. $6 \mathrm{a}$ and $6 \mathrm{~b}$ show spanwise pressure distributions for two post-stall angles of attack ( $\alpha=25^{\circ}$ and $33^{\circ}$ respectively) for both configurations. At the lower angle, control is effective across the entire span for the standard configuration, but at the higher angle control is less effective inboard and only maintains some authority outboard in the tip region (cf. fig. 4a). With sweep, control is ineffective near the tip, irrespective of $\alpha$. However, at high angles of attack control is particularly effective inboard with $\Delta C_{l} \approx 1$ near the root of the wing. Pressure distributions inboard $(y / s=0.17)$ and at the tip $(y / s=0.99)$ corresponding to these conditions for both configurations are shown in figs. 7a$7 \mathrm{~d}$ and $8 \mathrm{a}-8 \mathrm{~d}$. The inboard baseline pressure distribution shows leading-edge stall and the effect of control (fig. 7a) is similar to that observed on a similar airfoil. [10]. However, the swept-wing pressure distribution is significantly different and suggests the existence of a vortex generated at the leading-edge (fig. 7b), much like that of a delta wing, that is enhanced (fig. 7b) or generated (fig. 8b) when control is applied (see section 3.3). At the unswept tip, the existing tip vortex is strengthened at low and high $\alpha$, while virtually no effect is detectable at the wingtip with sweep at any $\alpha$ (figs. $7 \mathrm{~d}$ and $8 \mathrm{~d}$ ).
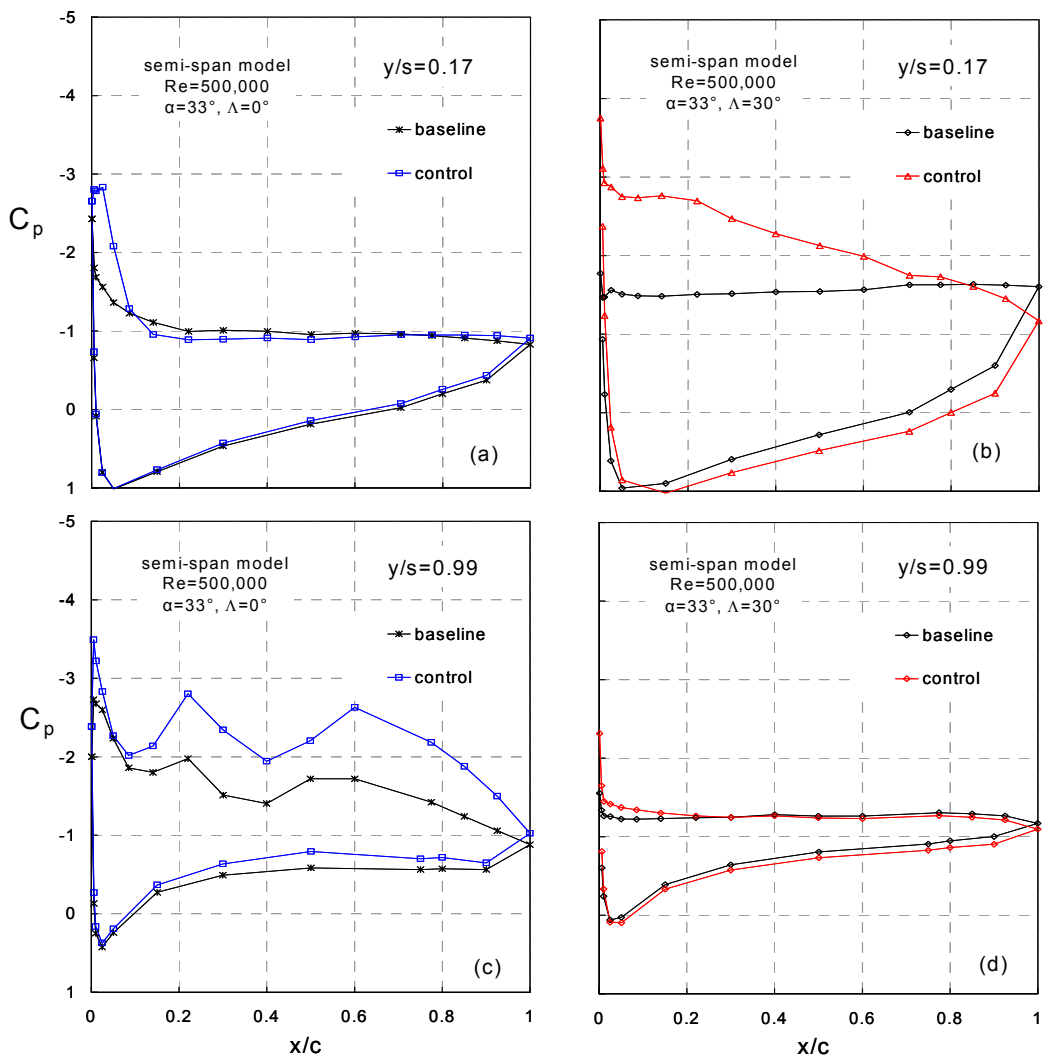

Fig. 8. Baseline and controlled pressure coefficient distributions at selected locations on the rectangular section of the model for standard and swept configurations ( $\alpha=33^{\circ}$; no wingtip extension).

The addition of a parallel tip (see schematic in fig. 2c) has a beneficial effect on the baseline lift (fig. 9), and particularly the lift generated in the vicinity of the wingtip (figs. 10a and 10b). The parallel tip increases the wing aspect ratio, and this results in a general increase in lift noted across the entire wingspan. More significantly, the parallel tip renders the original outboard pressure ports further inboard and this is reflected as significantly increased lift near the tip, although the additional lift generated by the wingtip has not been taken into account here. Despite the enhanced baseline lift, improvements to control effectiveness, measured in terms $\Delta C_{L}$ for each case considered individually, is negligible (fig. 9). It is further evident from figs. 10a and $10 \mathrm{~b}$ that the increment in $C_{l}$ across the entire span resulting from control is virtually unchanged as a result of the parallel tip. 


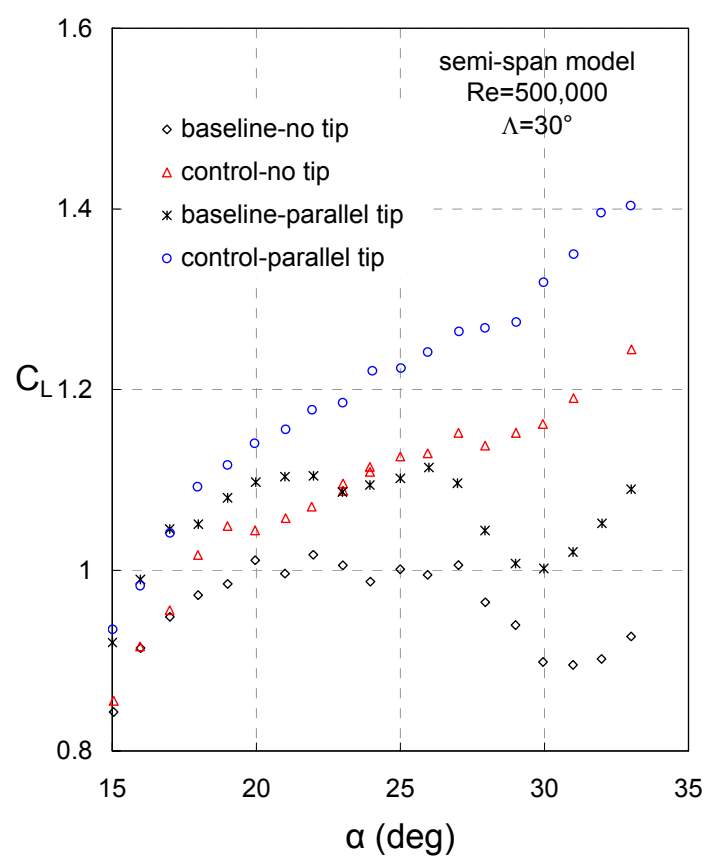

Fig. 9. Wing lift coefficient on the swept configuration, showing the effect of the parallel wingtip on leadingedge control effectiveness (control at $F^{+}=0.65$ and $C_{\mu}=0.3 \%$ ).

\section{Sweep Relations Applied to Control}

In considering control effectiveness on a swept airfoil that approximated infinite span, [2] it was noted that performance benefits were similar to the unswept case providing that the flow normal to the wing was considered. Thus the re-circulating region that results from leading-edge control on a NACA 0015 airfoil, in a time-mean sense, can also be expected to be present in the swept case. It should be expected, however, that the velocity component tangential to the leading-edge (axial velocity with respect to the vortex) has a stabilizing effect on the vortex. Furthermore, we assume that the control perturbations generated at the leading-edge are amplified and are convected downstream, normal to the leading-edge, much like their two-dimensional counterparts $[10,12]$. In the presence of sweep, however, there is a component of velocity tangential to the wing leading-edge, namely

$$
U_{\infty, t}=U_{\infty} \sin \Lambda^{\prime} .
$$

Therefore the perturbation will have a chordwise as well as a spanwise component. On a NACA 0015 airfoil (e.g. $[11,12])$, the fundamental perturbation phase velocity can be quantified to first approximation by the relationship:

$$
U_{\phi} / U_{\infty}=k(x / c)^{r}
$$

where $k$ and $r$ are constants that depend upon the separation control reduced frequency $F^{+}$(see fig. 11). Thus, to a first approximation, we assume that a similar relationship holds for flow normal to the swept wing, thus:

$$
U_{\phi, n} / U_{\infty, n}=k(x / c)^{r}
$$

where $k$ and $r$ now depend on the reduced frequency $F^{+}$defined normal to the leading-edge, $F_{n}^{+}$(see eqn. 5).

From equations (2), (6) and (8), the speed of the perturbation can be expressed as

$$
U_{\phi} / U_{\infty}=\sqrt{k^{2}(x / c)^{2 r} \cos ^{2} \Lambda^{\prime}+\sin ^{2} \Lambda^{\prime}}
$$

and its trajectory or "streamline" can be expressed as:

$$
\varepsilon_{\phi}=\sqrt{\tan \Lambda^{\prime} / k(x / c)^{r}}
$$


Airfoil data is shown in fig. 11 for two reduced frequencies with the same perturbation amplitude.
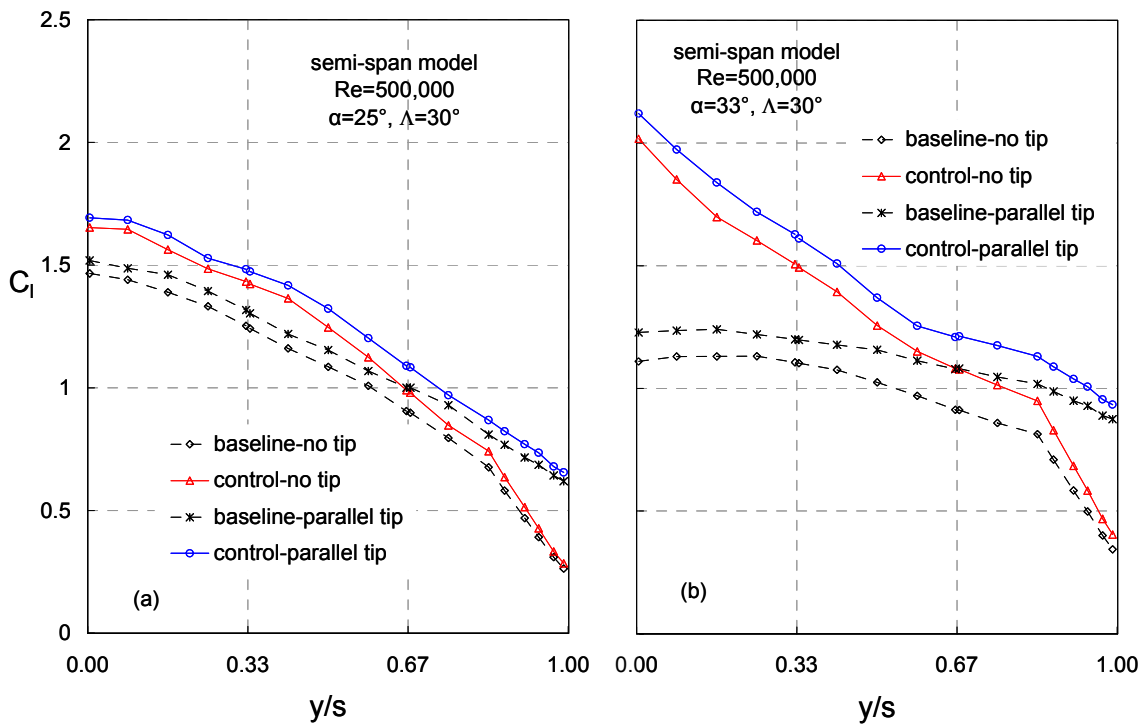

Fig. 10. Lift coefficient distributions on the swept configuration, showing the effect of the parallel wingtip on leading-edge control effectiveness ( $\operatorname{control}$ at $F^{+}=0.65$ and $C_{\mu}=0.3 \%$ ).

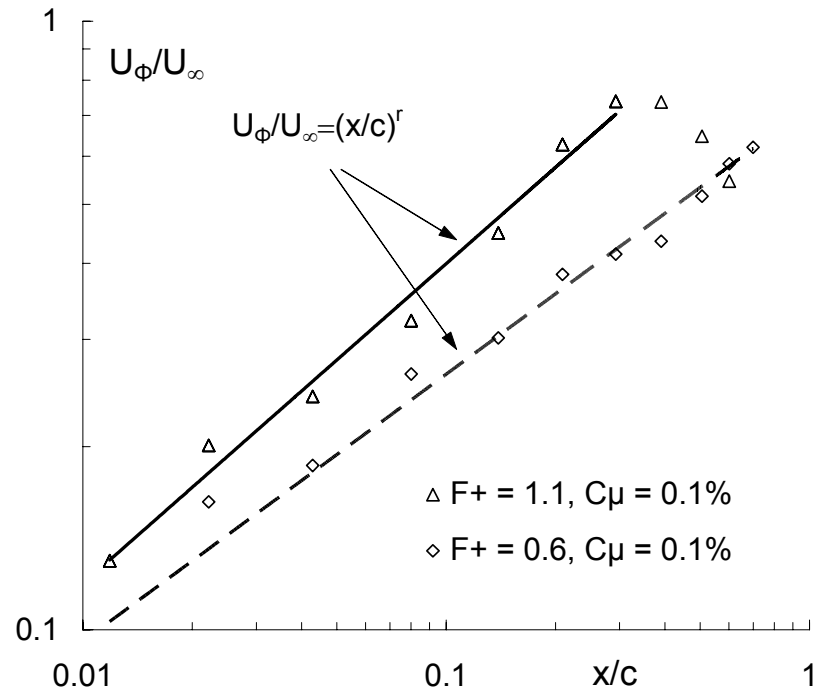

Fig. 11. Fundamental components of the phase-velocity measured on a NACA 0015 airfoil. [11]

By using empirical airfoil data to determine constants $k$ and $r$ at the control conditions $F^{+}=0.6$ and $C_{\mu}=0.1 \%$ (fig. 11), trajectories for different sweep angles were calculated (see fig. 12). The figure shows how the perturbation trajectories are swept across the span as a result of the tangential velocity component. Thus perturbations introduced near the tip will be swept off the wing without producing any meaningful change to the aerodynamic loads. Furthermore, as the sweep angle increases, the tangential velocity component increases and the effect is expected to diminish even more.

The above description attempts to provide a basic explanation for the gradually decreasing effect of control along the span and its total ineffectiveness at the tip. It can be seen from fig. 11 that, at $F^{+} \approx 1$, the approximation expressed in eqn. 7 breaks down at some point around $x / c \approx 0.3$. At larger $x / c$ the phase velocity tends towards a constant value, 
typically around $U_{\phi} / U_{\infty}=0.5$ to 0.6 . Taking this into account is indicated by the dashed hatched line on fig. 12 and shows that the assumption of eqn. 7 underestimates the deleterious effects of sweep on tip flow separation control at higher $F^{+}$.

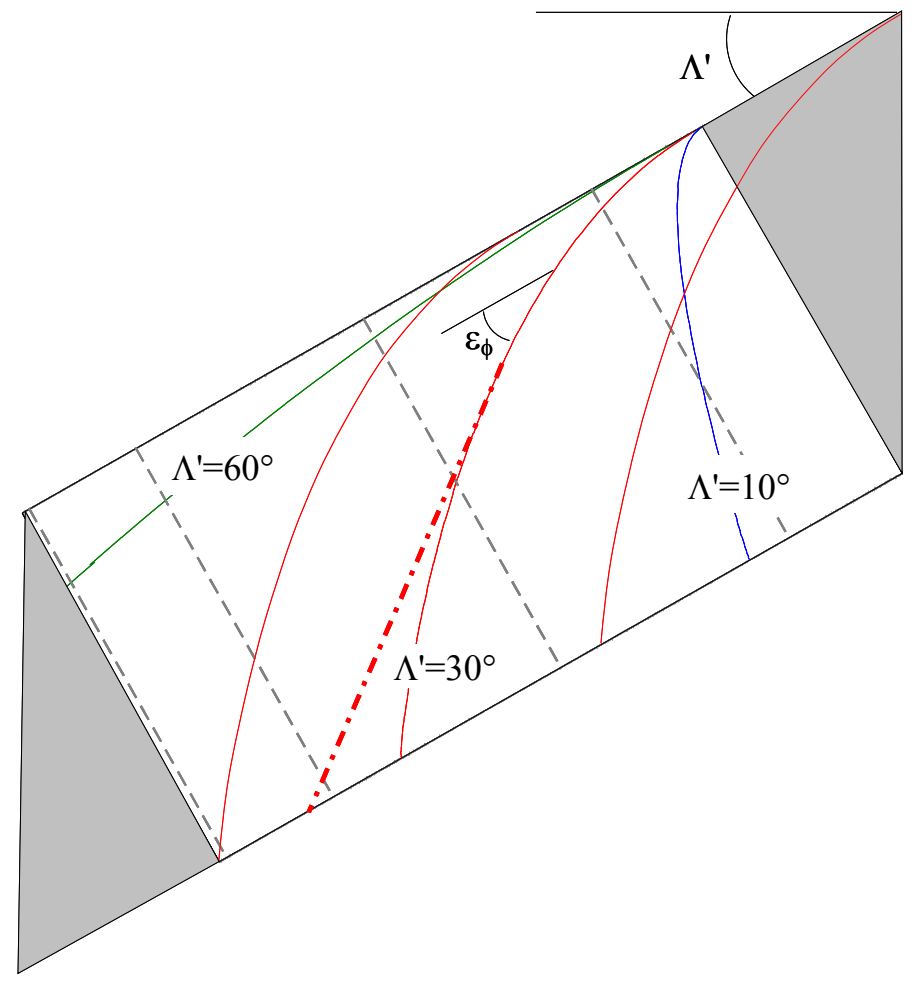

Fig. 12. Semi-empirical prediction of the fundamental component of the phase-velocity on a swept NACA 0015 wing based on data from fig. $11\left(F^{+}=0.65\right.$ and $\left.C_{\mu}=0.1 \%\right)$.

\section{Effect of Varying Free-Stream Velocity}

The attachment or separation of a shear layer to or from a vehicle wing is generally accompanied by a change in vehicle speed. In the former case, initiating active flow on a stalled wing, will generally increase lift, reduce drag and thereby result in acceleration of the vehicle. In the latter case, for example a sharp change to in attack on a controlled wing, with accompanying increase in drag, will result in a deceleration of the vehicle. Quasi-steady simulations of speed changes were simulated in the wind tunnel by changing the tunnel flow speed, in the range $10^{5} \leq R e \leq 10^{6}$, for standard and swept configurations at a large post-stall angle of attack, $\alpha=33^{\circ}$. Control was applied at $f=55 \mathrm{~Hz}$ and $U_{p}=17 \mathrm{~m} / \mathrm{s}(46.7 \mathrm{~Hz}$ and $15 \mathrm{~m} / \mathrm{s}$ for the swept case), in order to maintain the reduced frequency within the range considered to be effective $\left(0.3 \leq F^{+} \leq 2.6\right)$ and the perturbation amplitude corresponded to $0.02 \% \leq C_{\mu} \leq 2 \%$.

Lift and moment coefficient data is shown for standard baseline and control cases, where the flow speed, or Reynolds number, is initially decreased from $R e=10^{6}$ to $10^{5}$ and subsequently increased to $R e=10^{6}$ (figs. $13 \mathrm{a}$ and $13 \mathrm{~b}$ show outboard, $y / s=0.83$, and overall wing lift and moment coefficients). Two data points were acquired at each $R e$ based on a 15s average for each data point. The baseline data shows that as the velocity changes, the flow over the wing is bi-stable, i.e. either partially attached for fully separated. This can produce relatively large changes in the coefficients either at a constant speed or for relatively small changes in velocity. The bi-stable character observed between $R e=0.6 \times 10^{6}$ and $0.7 \times 10^{6}$ for both increasing and decreasing $R e$, produces a strong hysteresis effect. In contrast, control eliminates the bi-stable characteristic and consequently also eliminates hysteresis as a function of $R e$. Form drag exhibited similar behavior where $L / D p$ was typically between 1.3 and 2 .

The identical exercise to that described above was performed on the swept configuration (figs. $13 \mathrm{c}$ and $13 \mathrm{~d}$ show inboard, $y / s=0.83$, and overall wing lift and moment coefficients). With the introduction of sweep, the bi-stable flow associated with changing velocity in the baseline case is eliminated and the coefficients are virtually independent of 
the changing velocity. This difference between the post-stall baseline flows of the two configurations is consistent with the $C_{L}$ versus $\alpha$ data shown in figs. 3a and $3 \mathrm{~b}$. With control, the stable character is maintained and lift is enhanced, particularly inboard (fig. 13c). In addition, despite the relatively large increases in $C_{\mu}$ at low velocities, maximum inboard lift enhancement is achieved at $F^{+} \approx 0.6$; this is consistent the present unswept data as well as airfoil studies. [10,12]

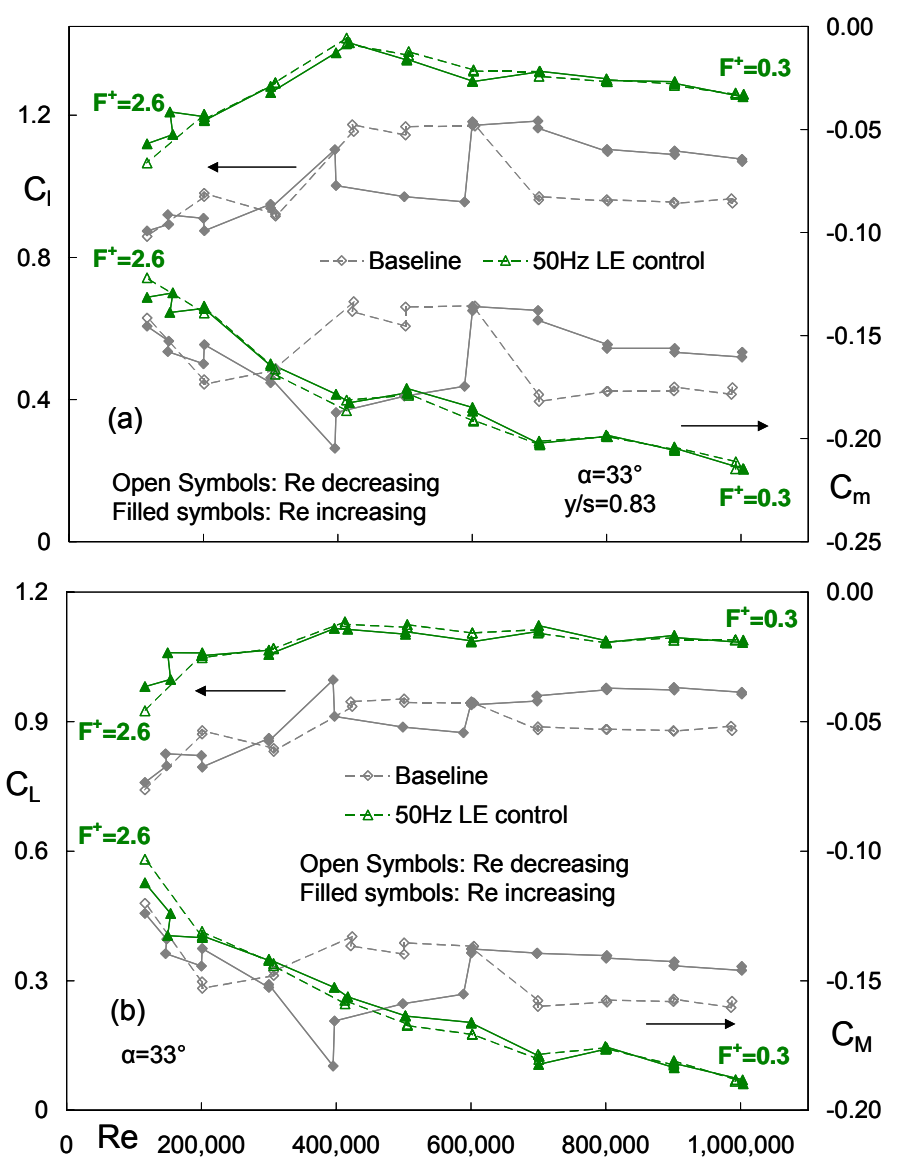

Fig. 13. Effect of changing free-stream velocity on the baseline and controlled standard model lift and moment coefficients (a) near the wingtip and (b) of the wing. Control at a constant frequency of $50 \mathrm{~Hz}$ and $U_{P}=17 \mathrm{~m} / \mathrm{s}$.

The favorable response of the flow to a range of reduced frequencies, rather than a single frequency, was exploited here by selecting the control frequency a priori to produce $0.3 \leq F^{+} \leq 2.6$. Thus, providing that the minimum threshold perturbation amplitude is exceeded, a fixed frequency can be used to effect control for a range of Reynolds numbers varying approximately by one order of magnitude. The effectiveness of control near the unswept wingtip does not diminish between $\alpha=25^{\circ}$ and $33^{\circ}$ (cf. figs. 6a and 6b). A similar conclusion can be drawn with respect to inboard control on the swept configuration, which in fact becomes more effective at higher $\alpha$. It is therefore reasonable to assume that control effectiveness in the standard configuration tip and swept inboard regions will be effective to even higher angles of attack, although this could not be verified due to limitations associated with the experimental setup.

The effectiveness of control near the tip, but not inboard, on the standard configuration is mostly due to enhancement of the tip vortex strength. In section 3.2.2 below, management of the vortex over a deflected tip flap (or aileron surrogate) is used to significantly enhance the wing moments. On the other hand, effectiveness of control inboard with sweep is presumed to be due to stabilization of the leading-edge vortex by the tangential (vortex-axial) flow. Nevertheless, based on the above discussion, we conclude that leading-edge active flow control at a single 
frequency can in principle be employed for vehicle control at very high $\alpha$ and at a range of speeds. One possible application may be to alleviate buffet and hysteresis on tilt-wing aircraft during the critical transition from vertical to horizontal flight as well a managing the so-called "barn door effect" during hover. [13,14]
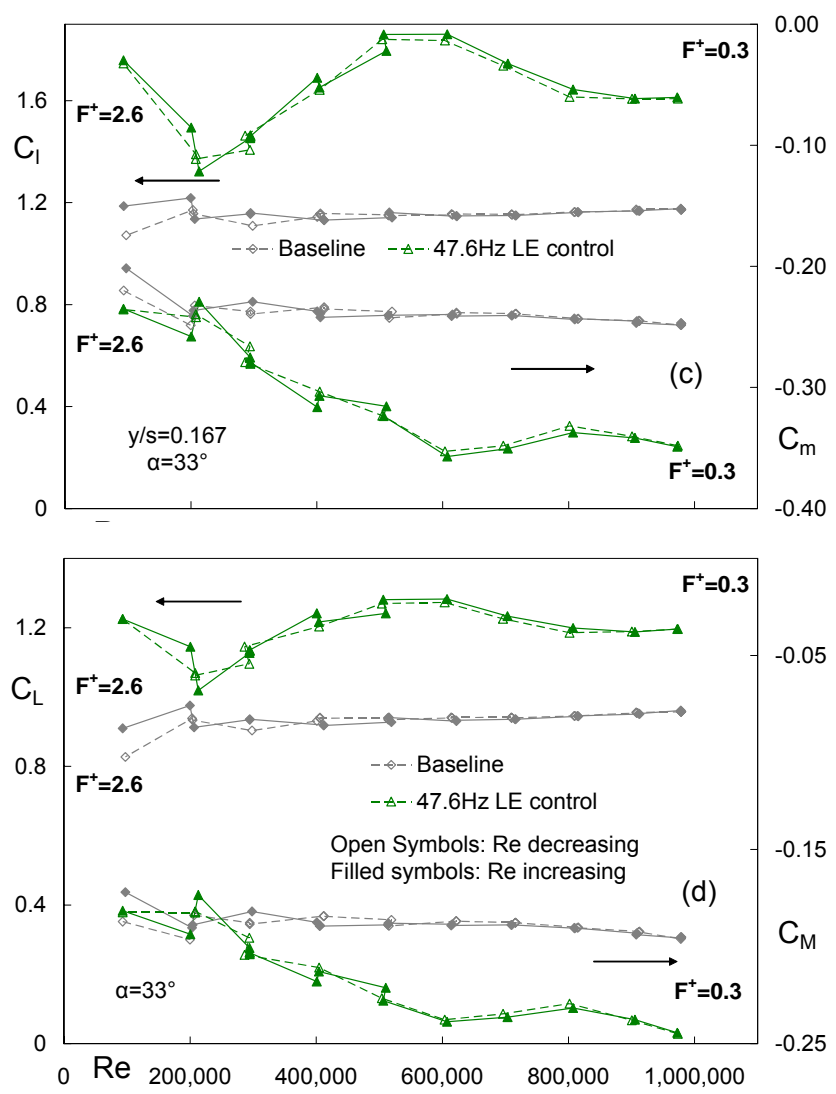

Fig. 13. Effect of changing free-stream velocity on baseline and controlled swept model lift and moment coefficients (c) inboard and (d) of the wing. Control at a constant frequency of $47.6 \mathrm{~Hz}$ and $U_{P}=15 \mathrm{~m} / \mathrm{s}$.

\section{Leading-edge Control with Flap Deflection}

Data for $\delta=\left(20^{\circ}, 20^{\circ}, 20^{\circ}\right)$ and $\delta=\left(40^{\circ}, 40^{\circ}, 40^{\circ}\right)$ flap deflections are presented in figs. 14a and $14 \mathrm{~b}$, where control is supplied from the leading-edge. For all leading-edge control data, the gap between the inboard edge of the inboard flap and the wind tunnel wall was sealed. At $20^{\circ}$ flap deflection, the flow over the flap appears to be partially attached up to $\alpha \approx 1^{\circ}$ for both swept and unswept configurations; at $40^{\circ}$ flap deflection the flap is stalled throughout the range of $\alpha$ considered here. Table 2 shows the changes to $\Delta C_{L, \max }$ as a result of leading-edge control combined with flap deflection. In both standard and swept configurations, $\Delta C_{L, \max }$ diminished as a result of increasing flap deflections, although clearly $C_{L, \max }$ increases. Thus flap deflection has a mild deleterious effect on leading-edge control effectiveness. Nevertheless, in a similar fashion to the symmetric wing case (no flap deflection), the majority of the lift enhancement is in the vicinity of the wing root while lift enhancement near the tip is negligible (figs. 15a and $15 \mathrm{~b}$ ) and the chordwise pressure distributions are similar (not shown).

Table 2. Changes in maximum lift coefficient $\left(\Delta C_{L, \max }\right)$ resulting from a combination of leading-edge control and flap deflections.

\begin{tabular}{|c|c||c|c|}
\hline$\left(\delta_{i}, \delta_{o}, \delta_{t}\right)$ & standard & Swept & tip configuration \\
\hline \hline$\left(0^{\circ}, 0^{\circ}, 0^{\circ}\right)$ & $\sim 0.24$ & $>0.23$ & no extension \\
\hline$\left(20^{\circ}, 20^{\circ}, 20^{\circ}\right)$ & $\sim 0.12$ & $\sim 0.20$ & no extension \\
\hline$\left(40^{\circ}, 40^{\circ}, 40^{\circ}\right)$ & $\sim 0.10$ & $\sim 0.13$ & no extension \\
\hline
\end{tabular}



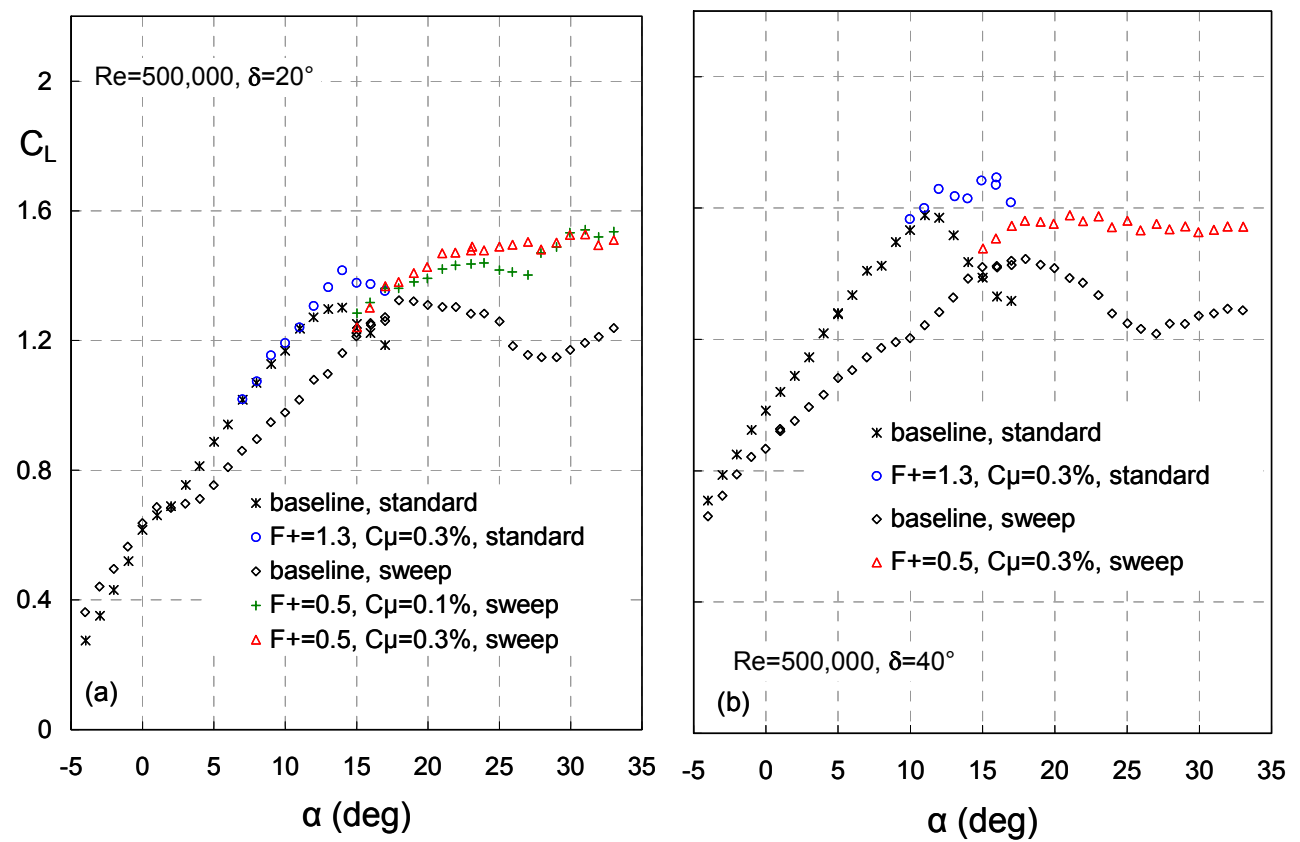

Fig. 14. The effect of control from the leading-edge in the presence of flap deflection (a) $\delta=\left(20^{\circ}, 20^{\circ}, 20^{\circ}\right)$ and (b) $\delta=\left(40^{\circ}, 40^{\circ}, 40^{\circ}\right)$.
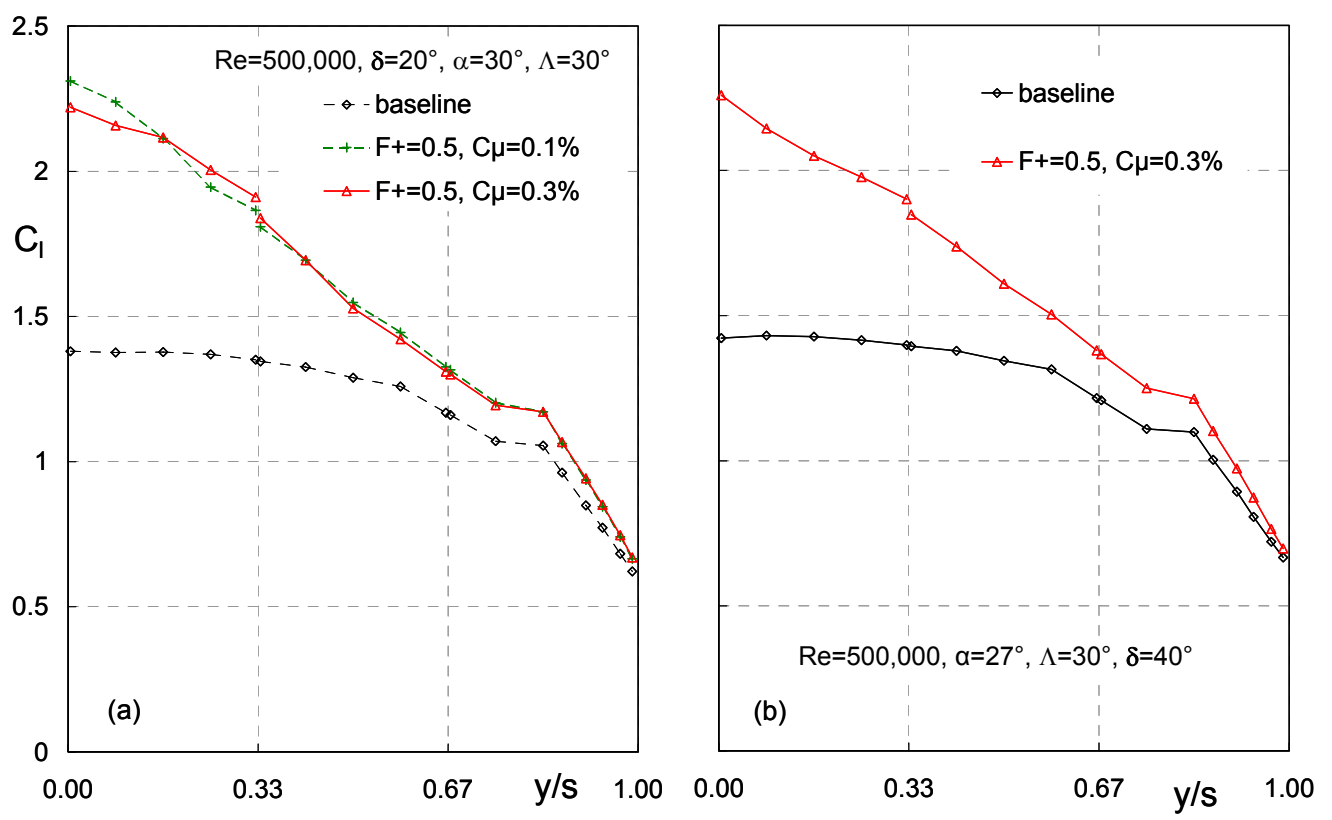

Fig. 15. Spanwise load variation for leading-edge control in the presence of flap deflection (a) $\delta=\left(20^{\circ}, \mathbf{2 0}^{\circ}, \mathbf{2 0}^{\circ}\right)$ and (b) $\delta=\left(40^{\circ}, 40^{\circ}, 40^{\circ}\right)$.

\section{B. Control from the Flap Shoulder}

1. Finite Flap Length

The effect of control in the presence of a finite flap span was assessed by comparing control on individually deflected flaps with control over the entire wingspan, $\delta=\left(20^{\circ}, 20^{\circ}, 20^{\circ}\right)$. The specific flap deflections considered were: (i) inboard and outboard flaps simultaneously $\delta=\left(20^{\circ}, 20^{\circ}, 0^{\circ}\right)$; (ii) the inboard flap $\delta=\left(20^{\circ}, 0^{\circ}, 0^{\circ}\right)$; and (iii) the 
outboard flap $\delta=\left(0^{\circ}, 20^{\circ}, 0^{\circ}\right)$; figs. 16a-16h. In the above figures depicting the span-load variation $\left(C_{l}\right.$ vs. $\left.y / s\right)$, data set pairs depict baseline and control cases respectively; in all cases the wingtip extension was not installed (cf. fig. 1a). All controlled flows were subjected to the same control perturbation frequencies and amplitudes along the span in order to facilitate an objective comparison between the various cases. It is evident from the data that control is effective across the span of each deflected flap. This can clearly be seen by comparing the $C_{l}$ near the flap edge and the adjacent un-deflected flaps for baseline and control cases $y / s=0.33$ and/or 0.67 in figs. $16 \mathrm{c}-16 \mathrm{~h}$ ). This has a significant effect on the flap trailing vortex properties as discussed in detail in ref. [15].
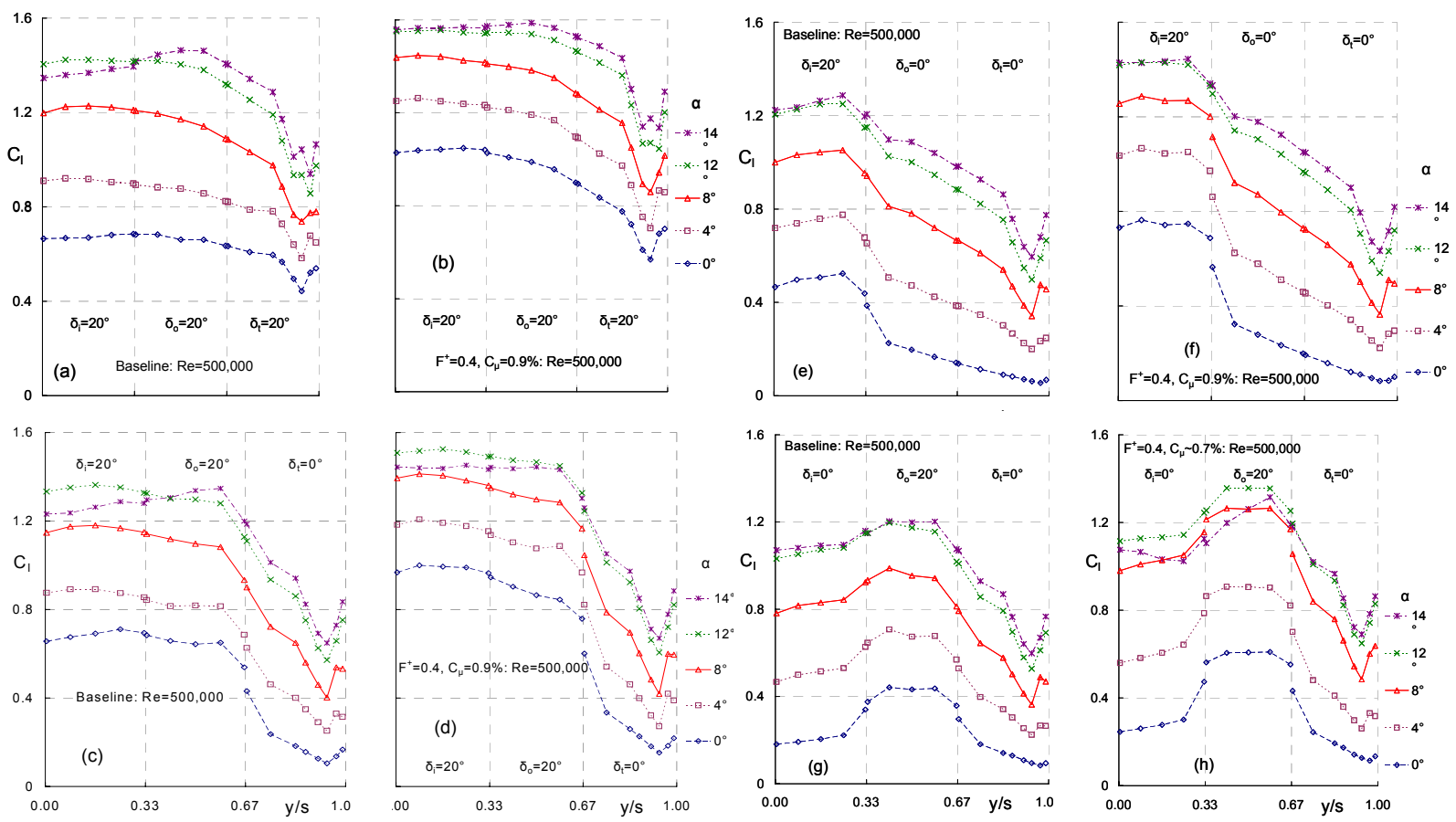

Fig. 16. Spanwise load variation showing the effect of control for different flap deflections, (a-b): full span; (cd): inboard and outboard; (e-f): inboard; (g-h) outboard.
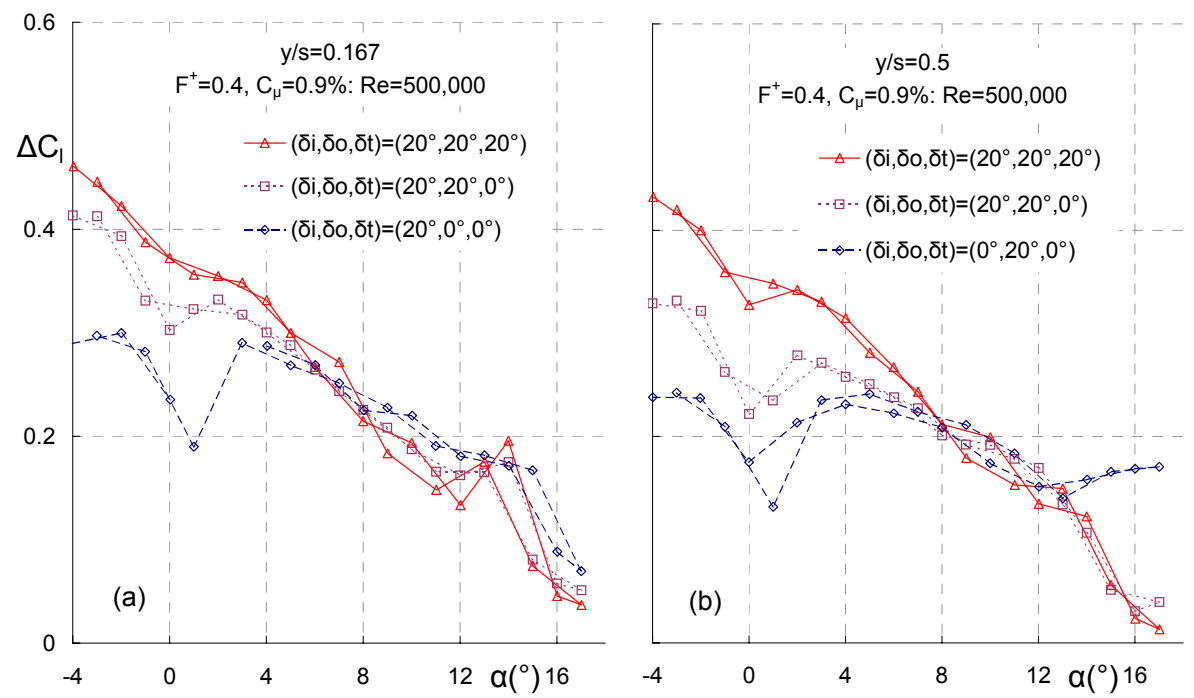

Fig. 17. Lift coefficient change with angle of attack (a) inboard and (b) at the midspan for different flap deflections. 
Successively shorter flap-span deflection, (i) to (iii) above, produces a successively narrower spanwise separated zone. The effect of this on control effectives is shown in fig. 17a where the local inboard $(y / s=0.167) C_{l}$ is plotted as a function of $\alpha$. At angles of attack less than $4^{\circ}$, the lift enhancement is greatest when all flaps are deflected and the effectiveness diminishes with successively narrower separated region. However for angles greater than approximately $6^{\circ}$ the differences in lift enhancement are small, and at $\alpha_{\max }$ there is a small but measurable switchover, with the shorter flap span producing more lift enhancement locally. Similar observations are made with respect to the outboard flap lift $(y / s=0.5$, fig. $17 \mathrm{~b})$, with the exception that control is clearly more effective on the deflected outboard flap $\delta=\left(0^{\circ}, 20^{\circ}, 0^{\circ}\right)$ at angles of attack exceeding approximately $12^{\circ}$.

A further effect of the narrower spanwise separated zone concerns control effectiveness along the flap span. In figs. $18 \mathrm{a}$ and $18 \mathrm{~b}$ the differences at the flap-edges are compared with those at the center span of the flap. At low angles of attack $\left(\alpha \leq 2^{\circ}\right)$ the effect of control is comparable between the flap centerline and edges; however, higher $C_{l}$ is generated at the outboard edge of the outboard flap for larger angles of attack. Inboard the differences are small with perhaps slightly larger lift enhancement near the wing/tunnel-wall junction (fig. 18b).
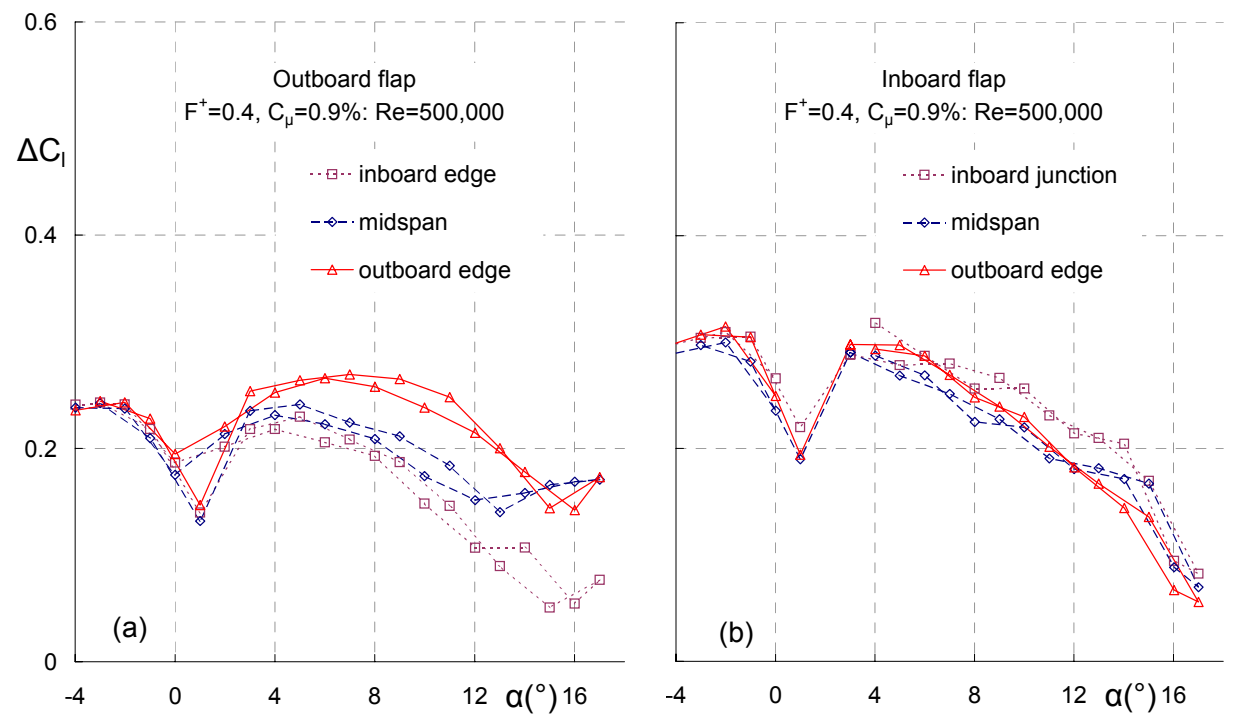

Fig. 18. Lift coefficient change with angle of attack at flap midspan and edges fir the (a) outboard and (b) inboard flaps.

\section{Tip Flap Control}

It was shown in section 3.1 that control remained effective at the wingtip even at large angles of attack. Here, control is introduced at the shoulder of the deflected tip flap $\delta=\left(0^{\circ}, 0^{\circ}, 20^{\circ}\right)$, with and without the square tip extension. The deflected tip flap can be thought of as an outboard control surface, such as an aileron. Thus, meaningful enhancement of the wing model moments can be expected to have direct implications for vehicle control and maneuverability.

Control of separation over the deflected tip flap depends strongly on the flap deflection angle, and also differs markedly from control on inboard flaps in many respects. For relatively small deflection angles $\left(\delta_{t}=20^{\circ}\right)$ control produces a local effect on the flap pressure distribution as shown at the flap centerspan (fig. 19a) and tip (fig. 19b). Unlike control applied inboard, or on a two-dimensional airfoil, there is virtually no influence on the forward $50 \%$ of the wing chord. This is so, even when the midspan pressure is consistent with that of a fully attached boundary layer, i.e. $C_{p}>1$ at the trailing-edge. However, with proximity to the wingtip, the effect on the flap pressure diminishes (fig. 19b). A further point to note is that relatively small $C_{\mu}$ are required to produce significant changes to the pressure distribution over the flap. For example, at $C_{\mu}=0.015 \%$, the pressure distribution is consistent with that that of a bubble enclosed on the upper surface, as characterized by the relatively low pressure downstream of the slot and subsequent pressure recovery with $C_{p}>1$ at the trailing-edge. [16] 
The net effect on lift across the entire wingspan is shown in fig. 20. As expected, the effect on lift is relatively small, and diminishes with angle of attack. The wingtip extension has the effect of slightly increasing the lift on the flap, under both baseline and controlled conditions, although a lift peak is observed at the tip when the extension is removed.
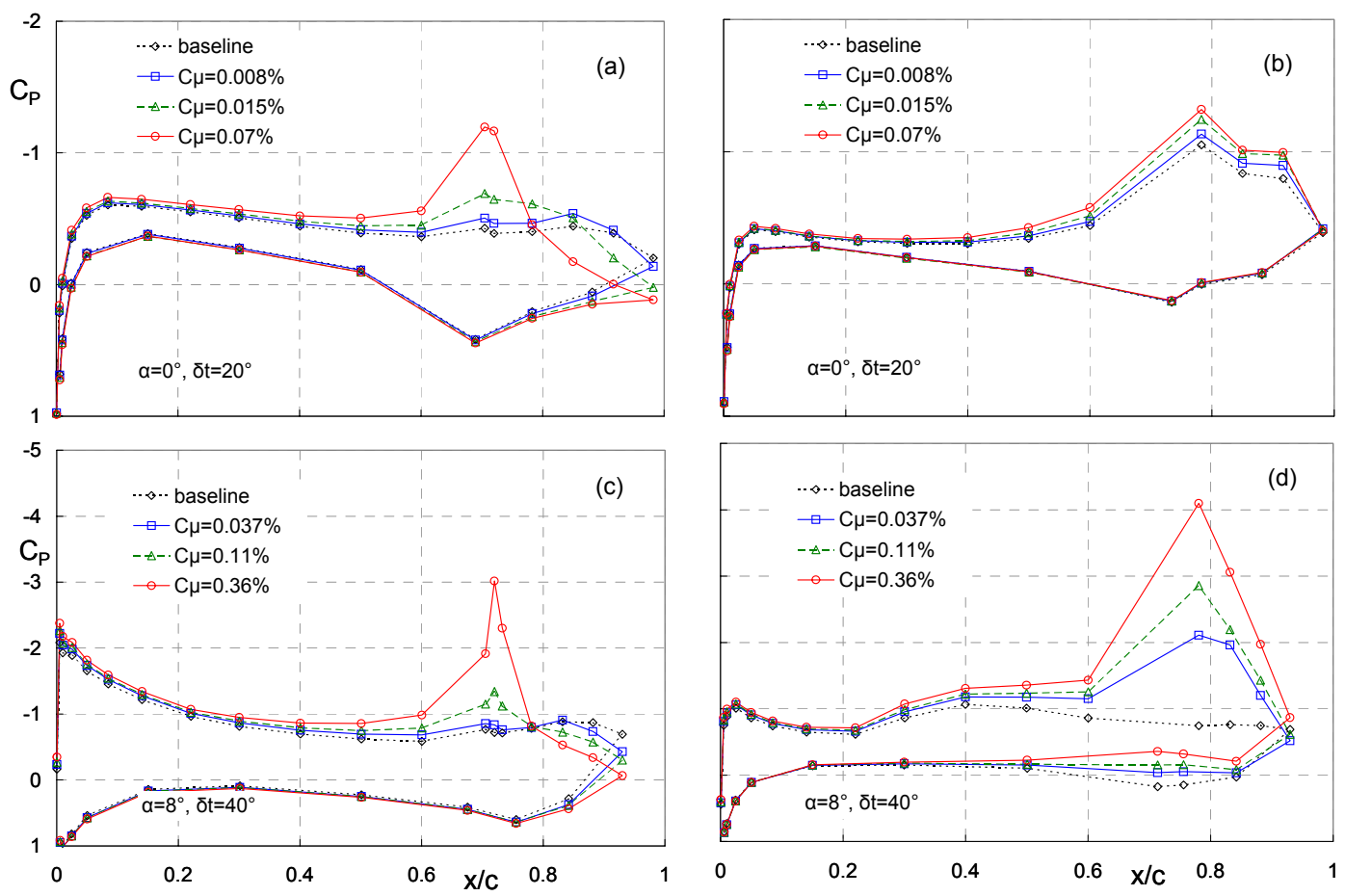

Fig. 19. Pressure distributions near the tip of the wing corresponding to control at the flap shoulder for (a-b) $20^{\circ}$ and (c-d) $40^{\circ}$ tip flap deflection.
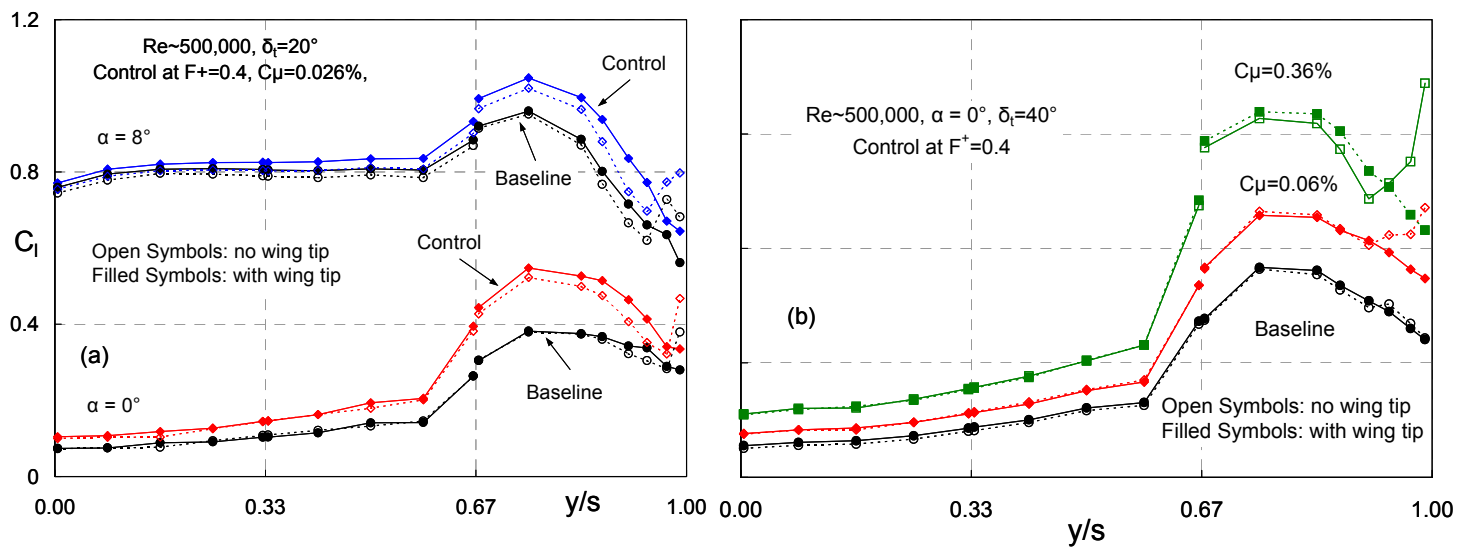

Fig. 20. Lift variation along the span for control at the tip flap shoulder for: (a) $20^{\circ}$ and (b) $40^{\circ}$ tip flap deflection.

Control in combination with a larger flap deflection $\left(\delta_{t}=40^{\circ}\right)$ produces a significantly greater effect (e.g. figs. $19 \mathrm{c}$ and 19d), although a larger $C_{\mu}$ is required to achieve this (note the different $C_{p}$ scaling). In stark contrast to the smaller deflection, the pressure over the flap at the tip is dramatically reduced, with $\Delta C_{p} \approx 3.4$ just downstream of the slot (fig, 19d). This is consistent with a larger and powerful tip vortex being partially rolled up on the flap; in this sense it represents an "on-demand" tip-flap vortex. Despite these relatively large changes to the pressure on the flap, 
and consistent with the $\delta_{t}=20^{\circ}$ case, the forward part of the wing chord corresponding to the defected flap remains virtually unaffected. Despite this, the local loads near the tip are significantly affected and the change in loading on the un-flapped inboard part of the wing is approximately constant along the span. The inboard portion of the flap extending to approximately the centerspan is uniformly affected producing $\Delta C_{l} \approx 0.4$, with and without the wingtip extension. Further outboard, without the extension, local $\Delta C_{l}$ drops and then increase dramatically towards the tip $\left(\Delta C_{l} \approx 0.7\right)$; with the extension $\Delta C_{l}$ diminishes slightly.
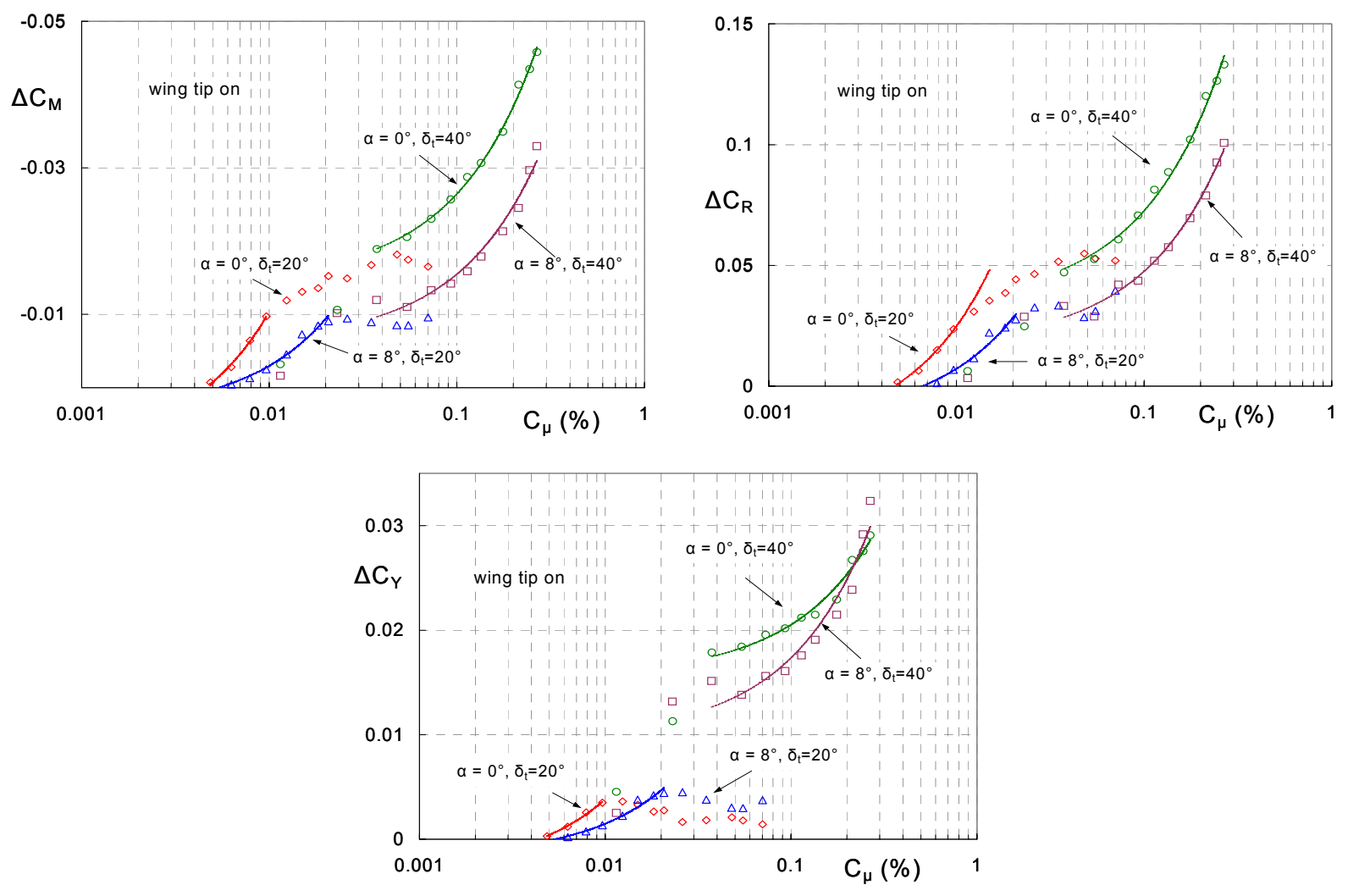

Fig. 21. (a) Pitching, (b) rolling and (c) yawing moment coefficients as a function of momentum coefficient for control at the tip flap shoulder (wing tip extension on).

The changes to pitching, rolling and yawing moment coefficients $\left(\Delta C_{M}, \Delta C_{R}\right.$, and $\left.\Delta C_{Y}\right)$ with the wingtip extension were assessed by integrating the surface pressures; results for fixed angles of attack $0^{\circ}$ and $8^{\circ}$ are shown for a fixed reduced frequency and increasing $C_{\mu}$ (figs. 21a-21c). Most quantitative trends associated with all three coefficients were similar and thus, apart from noted exceptions, the discussion below applies to all. Consistent with pressure data presented above, a relatively small control effect is observed on the moment coefficients at small flap deflection angles and the effect saturates at $C_{\mu} \approx 0.02 \%$. This is because the pressure distribution on the flap centerspan indicates an attached flow enclosing a bubble (see above) and further increases in $C_{\mu}$ serve to reduce the bubble length without markedly affecting the local lift. On the other hand, with larger flap deflection $\left(\delta_{t}=40^{\circ}\right)$, a larger separated shear layer is formed and larger $C_{\mu}$ brings the shear layer and accompanying tip vortex successively closer to the surface. The pressure distribution does not indicate a full pressure recovery for relatively large amplitudes and $C_{\mu}=0.36 \%$ is required to produce a pressure recovery that is consistent with that of an attached flow. Clearly, the larger control authority comes with a cost and this can be quantified by noting that, for the two different flap deflections, the ratio $d C_{R} / d C_{\mu}$, for example, is less than 0.1 .

Although greater authority could conceivably be achieved at the maximum flap deflection considered here by increasing $C_{\mu}$, it should be expected that control authority is close to saturation as the pressure recovery on the flap midspan is consistent with that of an attached, or nearly attached, boundary layer (fig. 17c). 
For both flap deflections, the dependence of all moment coefficients on $C_{\mu}$ is linear, providing $C_{\mu}$ exceeds some threshold and is less than that when control saturates. For $C_{M}$ and $C_{R}$, the control authority achieved at $\delta_{t}=20^{\circ}$ when control saturates, is comparable to that achieved with minimum threshold $C_{\mu}$ at $\delta_{t}=40^{\circ}$. This is not true for $C_{Y}$ where the authority jumps by approximately 0.1 for the two different flap deflections. This can be explained by considering the different components of the load on the flap. In the former cases $\left(C_{M}\right.$ and $\left.C_{R}\right)$, assuming the same load on the flap and no change to the wing load upstream of the control slot, the change in flap angle reduces the component contributing to the moments by approximately $18 \%\left(\cos \delta_{2}-\cos \delta_{1}\right) / \cos \delta_{1}$. However, in the case of $C_{Y}$, the change in flap angle increases the contributing component by approximately $88 \%\left[\left(\sin \delta_{2}-\sin \delta_{1}\right) / \sin \delta_{1}\right]$. It should therefore be expected that, providing the flow can be attached to the flap, larger flap deflections will produce diminishing authority over $C_{M}$ and $C_{R}$ and increasing authority over $C_{Y}$. For example, using the same reasoning as above, an increase of the flap deflection angle to $60^{\circ}$ will decrease $C_{M}$ and $C_{R}$ by $47 \%$ and increase $C_{Y}$ by $153 \%$. This is a simplified explanation because the load on the flap does in fact increase with larger controlled flap deflections. It should therefore be expected that large controlled flap deflections $\delta_{l}>40^{\circ}$, will produce moments well in excess of conventional control surfaces; yawing moment coefficients will be dramatically larger.

The linear dependence of the moment coefficients $C_{\mu}$ should be contrasted with flapped airfoils and inboard flaps, where two scenarios are generally observed. At small $\delta$ and $\alpha$, the flow attaches to the flap when some thresholds is reached and then control saturates with larger $C_{\mu}$ producing negligible performance increments. For large $\delta$ and/or $\alpha$, the changes to lift, for example, are typically logarithmic and $C_{\mu}>1 \%$ is generally required to attach the flow fully to the flap.

Similar results were obtained at different reduced frequencies in the range $0.3<F^{+}<2$ and with the wingtip extension removed (not shown). Moment coefficient data as a function of $\alpha$, with the wingtip extension removed, is shown for $\delta_{t}=40^{\circ}$ (figs. 22a-22c). The two control data sets correspond to forcing amplitudes just above the threshold necessary for linear control $\left(C_{\mu}=0.06 \%\right)$ and close to control saturation $\left(C_{\mu}=0.36 \%\right)$. Both produce a significant effect on moments throughout the $\alpha$ range: in the case of $C_{M}$ and $C_{R}$, the effect approximately doubles with a factor of six increase in $C_{\mu}$. In the case of $C_{Y}$, small $C_{\mu}$ produces a relatively large effect at low $\alpha$, and this effect diminished as $\alpha$ increases.
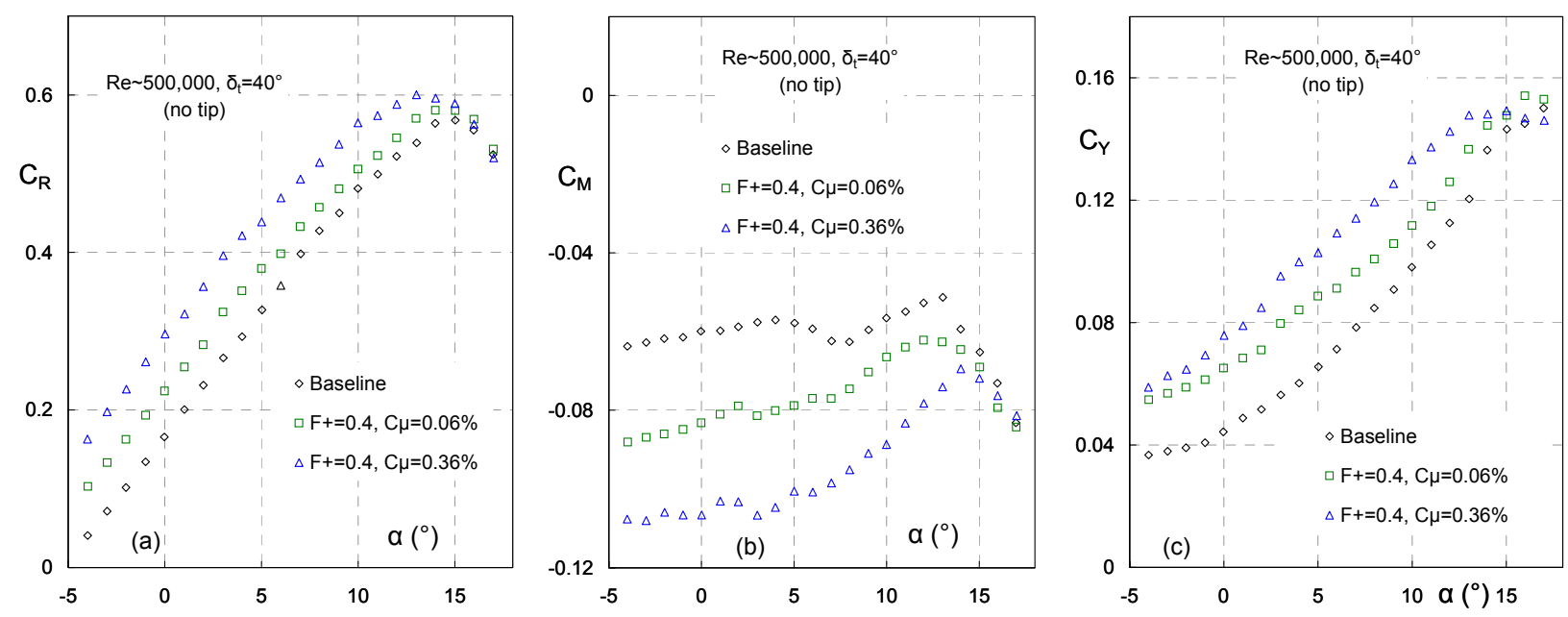

Fig. 22. (a) Pitching, (b) rolling and (c) yawing moment coefficients as a function of angle of attack for control at the tip flap shoulder (wingtip extension off).

\section{Flap-shoulder control with Sweep}

Data for control from the flap-shoulder are shown for both standard and swept configurations in figs. 23-25. For all data presented, flaps are deflected in unison, i.e. either $\delta=\left(20^{\circ}, 20^{\circ}, 20^{\circ}\right)$ or $\delta=\left(40^{\circ}, 40^{\circ}, 40^{\circ}\right)$. The incremental changes to $C_{L}$ associated with the standard configuration at $20^{\circ}$ flap deflection are not matched by those of the swept configuration (fig. 23a). In addition, the increase in $C_{L, \max }$ associated with the standard configuration is not matched by that of the swept configuration. At angles of attack greater than where the flap stalls $\left(\alpha>1^{\circ}\right)$ pressure coefficient 
distributions on the flap show slightly better recovery when sweep is introduced (e.g. figs. 24b-24c). This suggests that the flow on baseline swept configuration is less separated than that on the standard configuration. The reason for this is not clear, but it may be due to a stabilized vortical flow, resulting from the tangential velocity component at the flap-shoulder, and positioned over the flap. The pressure coefficients are based on the normal $U_{\infty, n}$ and the apparent enhanced attachment does not translate to higher lift. Much like in the case of leading-edge control, flapshoulder control appears to be more effective inboard for the swept configuration, while control effects near the tip are negligible; this is evident from the span-load variations (figs. 25a). Unlike the case of leading-edge control, flapshoulder control on the swept configuration does not produce larger increments inboard. As noted above, without sweep the effect of control is approximately uniform across the span (fig. 25a) and inboard its effect is approximately double that of the swept configuration. At present there is no clear explanation why sweep has such a deleterious effect on flap shoulder control.
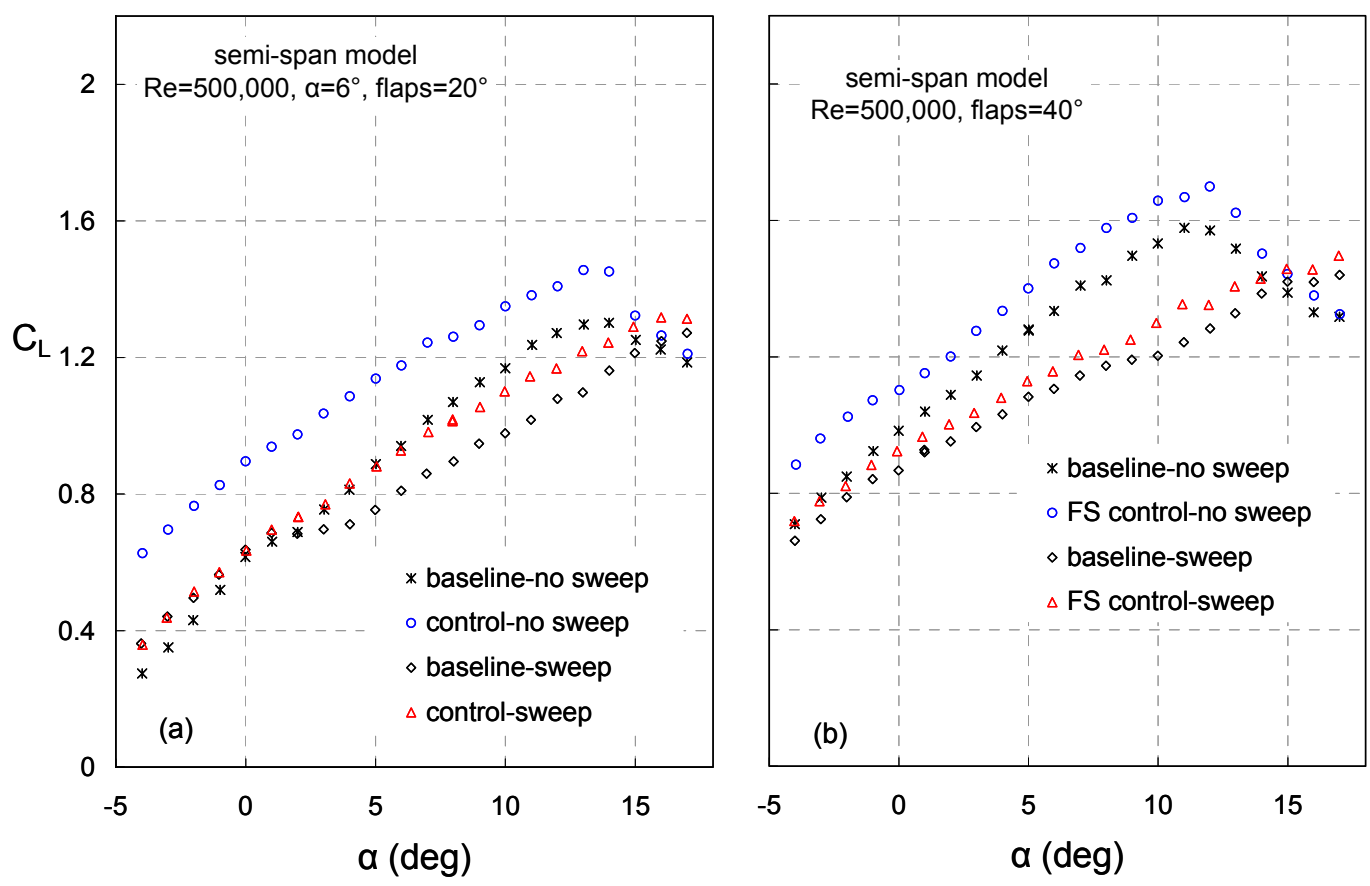

Fig. 23. Lift coefficient variation showing the effect of sweep on flap shoulder control for (a) $\delta=\left(20^{\circ}, \mathbf{2 0}^{\circ}, \mathbf{2 0}^{\circ}\right)$ and (b) $\delta=\left(40^{\circ}, 40^{\circ}, 40^{\circ}\right)$. All control at $F^{+}=0.4$ and $C_{\mu}=1.4 \%$.

At the large $40^{\circ}$ flap deflection the control amplitude is not large enough to significantly control the flap flow for either the standard or swept configurations. Nevertheless, the standard configuration is once again more receptive to control, with a relatively small but uniform lift enhancement across the span. When sweep is introduced, almost no effect is detectable at $y / s>0.3$. Similar results to this above were observed with individual flap deflections, such as those presented in section 3.2.1. It can therefore be concluded that flap shoulder control on low aspect ratio wings with substantial sweep (i.e. $\geq 30^{\circ}$ ), will not produce significant performance increments.

Figs. 26a and 26b simultaneously consider the effect of (i) the wingtip extension and (ii) the gap between the inboard edge of the inboard flap and the wind tunnel wall on the baseline flow. In the first case the flap-edge/windtunnel wall gap is left open and the wing is equipped with a square tip; in the second case this gap is sealed and no tip extension is present. The distance between the inboard and tip, and hence their negligible effect on one another, allows these effects to be studied simultaneously. For both baseline cases the flap stalls at $\alpha \approx 1^{\circ}$ but the case with the gap flow produced slightly more lift. The gap assists in promoting attachment of the flow to the flap and this can be seen by the larger inboard lift (fig. 26b). With control the lift is slightly higher, but the difference between this and the baseline case is smaller than the baseline versus control difference associated with the sealed case. The lift near the tip drops dramatically irrespective of the whether there is a tip extension or not. However, inclusion of the tip does result in slightly higher lift in that region and the control effectiveness is also marginally better. 

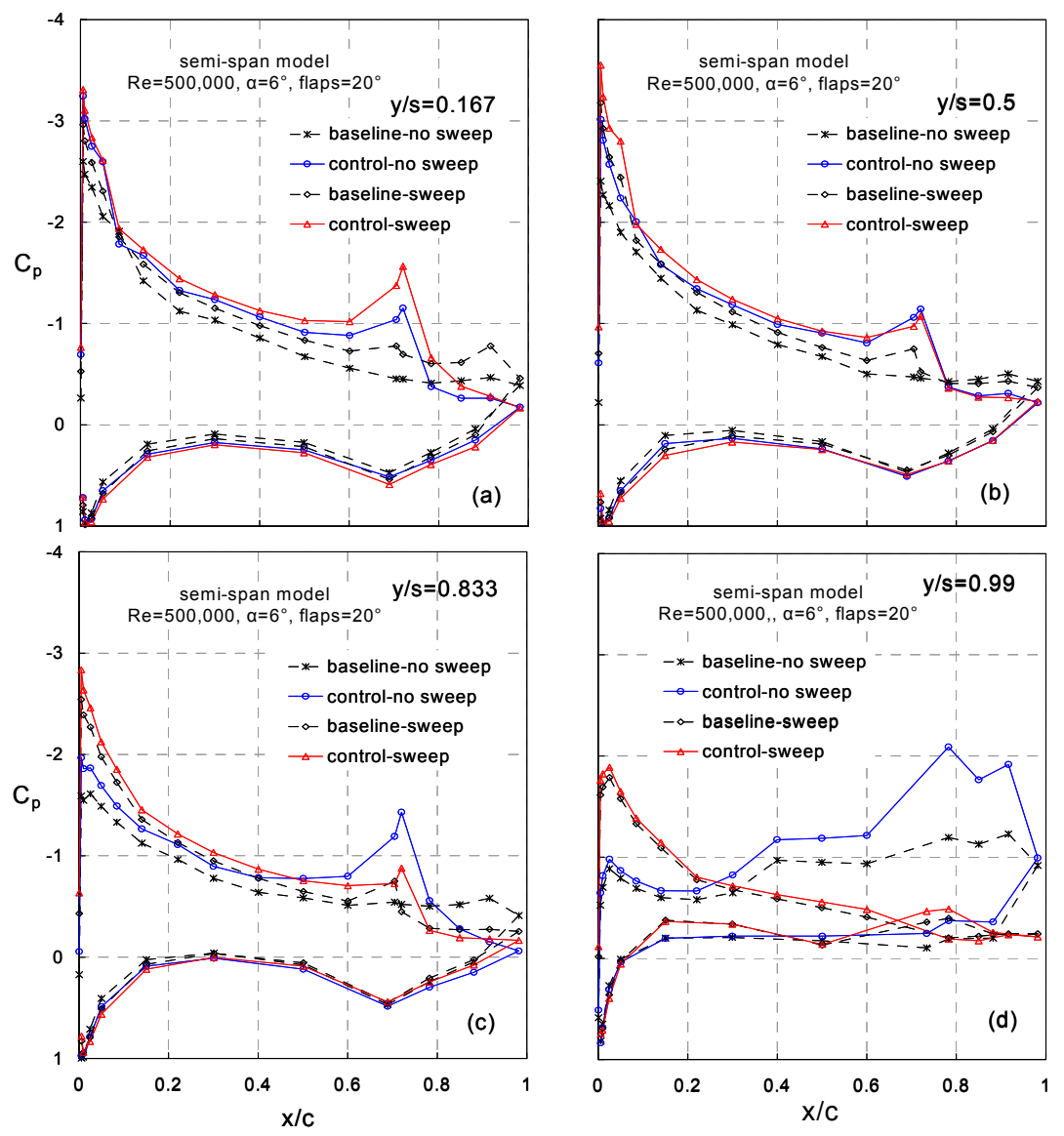

Fig. 24. Selected wing pressure coefficients showing the effect of sweep on flap shoulder control at $\delta=\left(20^{\circ}, 20^{\circ}, 20^{\circ}\right)$. All control at $F^{+}=0.4$ and $C_{\mu}=1.4 \%$.
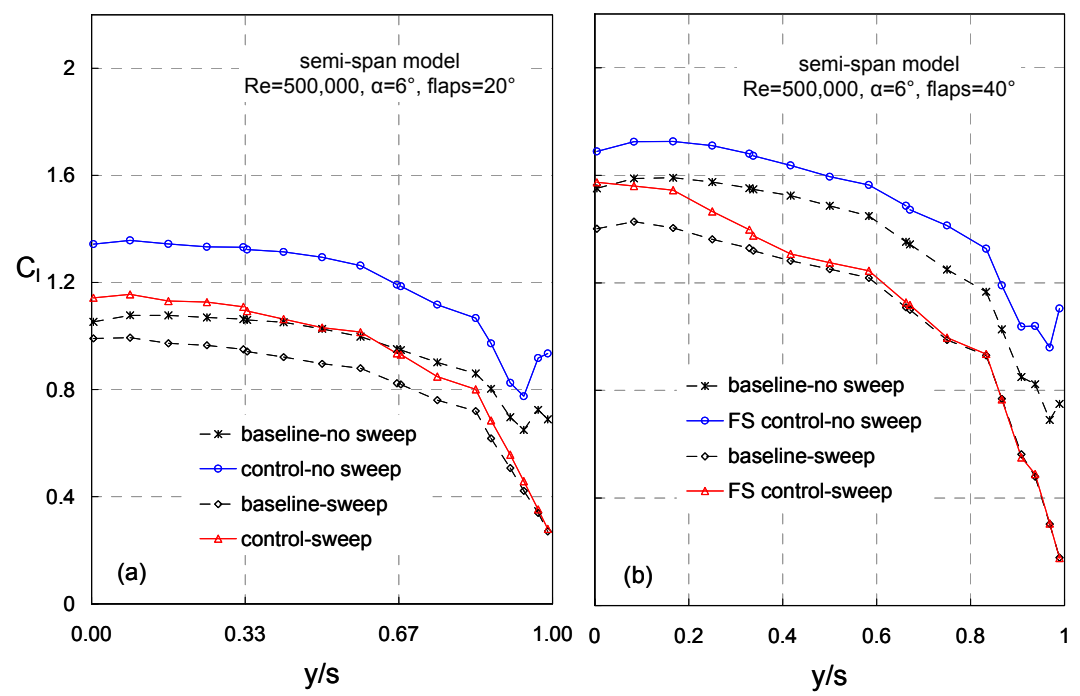

Fig. 25. Local lift variation showing the effect of sweep on flap shoulder control for (a) $\delta=\left(20^{\circ}, 20^{\circ}, 20^{\circ}\right)$ and (b) $\delta=\left(40^{\circ}, 40^{\circ}, 40^{\circ}\right)$. All control at $F^{+}=0.4$ and $C_{\mu}=1.4 \%$. 

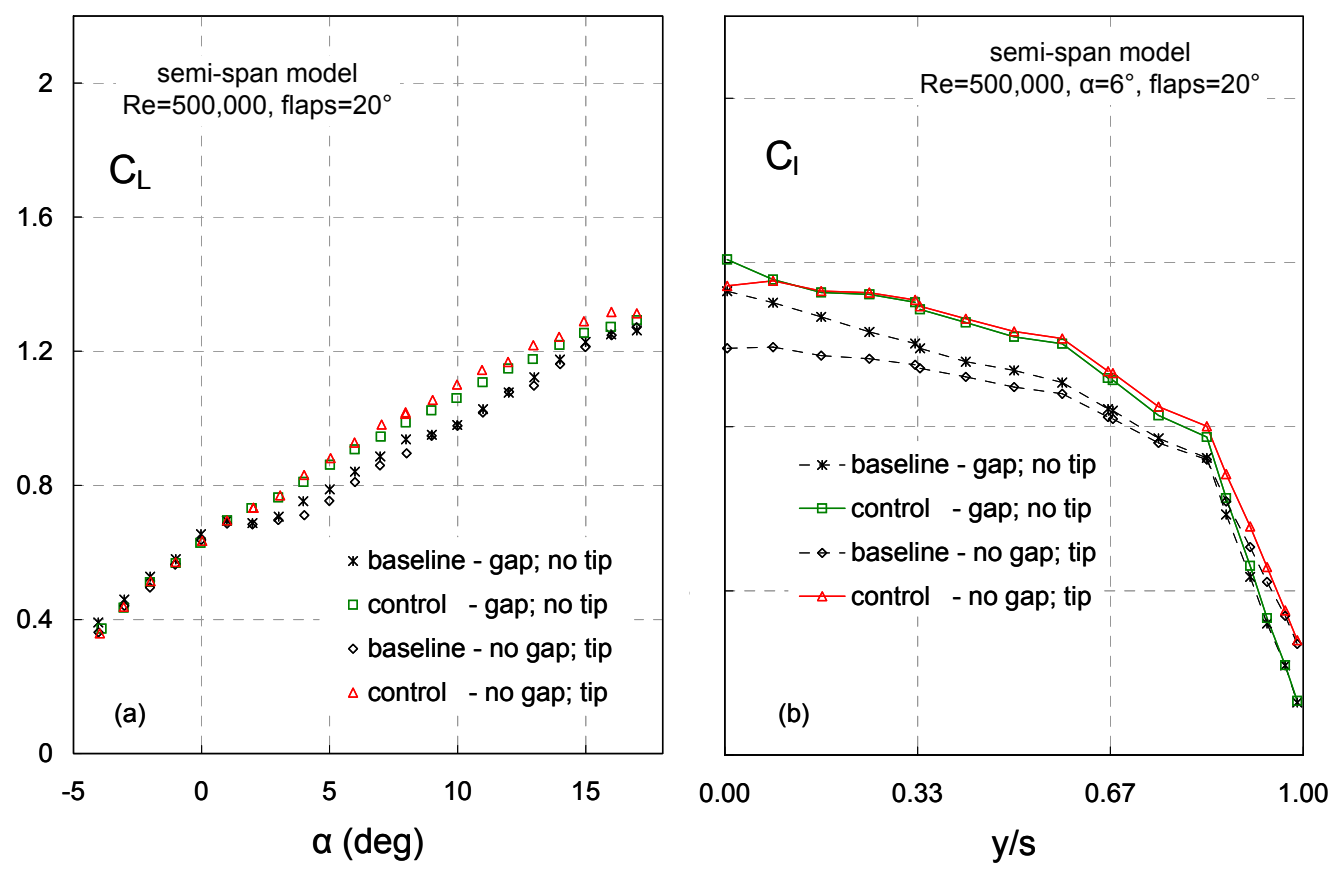

Fig. 26. Lift coefficient variation showing the effect of sweep on flap shoulder control in the presence of a wing/tunnel-wall gap and with a wingtip extension at $\delta=\left(20^{\circ}, 20^{\circ}, 20^{\circ}\right)$. All control at $F^{+}=0.4$ and $C_{\mu}=1.4 \%$.

\section{Summary of Main Findings}

The efficacy of separation control on the semispan wing was investigated by means of leading-edge and flapshoulder zero mass-flux blowing slots. Without sweep baseline stall was initiated inboard, but with sweep stall was initiated near the tip. Leading-edge perturbations were effective for increasing $C_{L, \max }$ and post stall lift. Without sweep, the effect of control was approximately uniform across the wing span and maintained authority near the tip at high $\alpha$; when sweep was introduced a significant effect was noted inboard, but this effect degraded along the span and produced virtually no meaningful lift enhancement near the tip, irrespective of the tip configuration. These basic trends were maintained in the presence of flap deflections of $20^{\circ}$ and $40^{\circ}$. A simple model, based on the trajectory or "streamline" of the perturbation, served to explain the poor performance of separation control near the wingtip.

Control on finite-span flaps did not differ significantly from their two-dimensional counterparts. Control over a tip flap produced significant moments in the presence of large deflection and the variations were linear with $C_{\mu}$. As expected, control from the flap produced meaningful lift enhancement and $C_{L, \max }$ improvements in the absence of sweep. However, with the introduction of sweep, these improvements were significantly degraded and changes to $C_{L, \text { max }}$ were insignificant.

A case can be made for leading-edge control applied to a wing with moderate sweep and/or high aspect ratio. Flapshoulder control on a swept wing should not be expected to produce effects comparable to those on unswept configurations.

\section{Acknowledgements}

This work was performed while the first author held a National Research Council-NASA Langley Research Center Associateship. The authors wish to thank W. L. Sellers III for initially suggesting and supporting the study of basic three-dimensional effects in the context of active flow control. The authors also wish to thank S. A. Gorton, C. S. Yao, L. P. Melton, L. N. Jenkins, I. Wygnanski (University of Arizona) and H. Nagib (Illinois Institute of Technology) for their active assistance and many fruitful discussions; as well as R. D. White, A. Barnes and J. Harris for their exceptional technical support. 


\section{References}

1. Greenblatt, D. and Wygnanski, I, "Control of separation by periodic excitation," Progress in Aerospace Sciences, Volume 37, Issue 7, pp. 487-545, 2000.

2. Naveh, T., Seifert, A., Tumin, A. and Wygnanski, I., "Sweep effect on parameters governing control of separation by periodic excitation", AIAA Journal, Vol. 35, No. 3, 1998, pp. 510-512.

3. Guy, Y., Morrow, J. and Mclaughlin, T., "Control of vortex breakdown on a delta wing by periodic blowing and suction" AIAA Paper 99-132, $37^{\text {th }}$ Aerospace Sciences Meeting and Exhibit, Reno, NV, Jan. 12-15, 1999.

4. Guy, Y., Morrow, J. and Mclaughlin, T., "Parametric investigation of the effects of active flow control on the normal force of a delta wing" AIAA Paper 2000-0549, 38 th Aerospace Sciences Meeting and Exhibit, Reno, NV, Jan. 12-15, 2000.

5. Margalit, S., Greenblatt, D., Seifert, A. and Wygnanski, I., "Delta wing stall and roll control using segmented piezoelectric fluidic actuators," AIAA Journal of Aircraft, Vol. 42, No. 3, 2005, pp. 698-709.

6. Amitay, M., Washburn A. E., Anders, S. G. and Parekh, D. E., "Active Flow Control on the Stingray Uninhabited Air Vehicle: Transient Behaviour," AIAA Journal, vol.42 no.11, 2004, pp. 2205-2215.

7. Greenblatt, D., "Dual location separation control," AIAA Paper 2005-5085, 23rd AIAA Applied Aerodynamics Conference, Westin Harbour Castle, Toronto, Ontario, 6-9 June 2005. (AIAA Journal, in press.)

8. Spivey, W. A. and Morehouse, G. G., "New insights into the design of swept-tip rotor blades," 26th National Annual Forum Proceedings of the American Helicopter Society, Washington, D.C., 1970.

9. McAlister, K. W. and Takahashi, R. K. "NASA 0015 wing pressure and trailing vortex measurements" NASA TP 3151 (AVSCOM Technical Report 91-A-003), November 1991.

10. Greenblatt, D. and Wygnanski, I., "Effect of leading-edge curvature on airfoil separation control," AIAA Journal of Aircraft, Vol. 40, No. 3, 2002, pp. 473-481.

11. Greenblatt, D., Nishri, B., Darabi, A. and Wygnanski, I., "Dynamic stall control by periodic excitation. Part 2: Mechanisms," AIAA Journal of Aircraft, Vol. 38, No. 3, 2001, pp. 439-447.

12. Seifert, A., Darabi, A. and Wygnanski, I., "Delay of airfoil stall by periodic excitation”, AIAA Journal of Aircraft, Vol. 33, No. 4, 1996, pp. 691-698.

13. Sullivan, T. M., "The Canadair CL-84 tilt wing design," AIAA paper 1993-3939, Aircraft Design, Systems and Operations Meeting, Monterey, CA, Aug 11-13, 1993.

14. Manley, D.J., and von Klein, Jr., W., "Design and development of a super-short takeoff and landing transport aircraft," AIAA Paper 2002-6023, 2002 Biennial International Powered Lift Conference and Exhibit, 5-7 November 2002, Williamsburg, VA.

15. Greenblatt, D., "Managing Flap Vortices via Separation Control," AIAA Journal, Vol. 44, No. 11, 2006, pp. $2755-2764$.

16. Greenblatt, D., Melton, L., Yao, C., Harris, J., "Control of a Wing Tip Vortex" AIAA Paper 2005-4851, 23rd AIAA Applied Aerodynamics Conference, Westin Harbour Castle, Toronto, Ontario, 6-9 June 2005. 